\title{
Generalized Darlington Synthesis
}

\author{
Patrick Dewilde, Fellow, IEEE
}

(Invited Paper)

In honor to the great scientist and engineer Sidney Darlington

\begin{abstract}
The existence of a "Darlington embedding" has been the topic of vigorous debate since the time of Darlington's original attempts at synthesizing a lossy input impedance through a lossless cascade of sections terminated in a unit resistor. This paper gives a survey of present insights in that existential question. In the first part it considers the multiport, time invariant case, and it gives the necessary and sufficient conditions for the existence of the Darlington embedding, namely that the matrix transfer scattering function considered must satisfy a special property of analyticity known as "pseudomeromorphic continuability" (of course aside from the contractivity condition which ensures lossiness). As a result, it is reasonably easy to construct passive impedances or scattering functions which do not possess a Darlington embedding, but they will not be rational, i.e. they will have infinite dimensional state spaces. The situation changes dramatically when time-varying systems are concerned. In this case also Darlington synthesis is possible and attractive, but the anomalous case where no synthesis is possible already occurs for systems with finite dimensional state spaces. We give precise conditions for the existence of the Darlington synthesis for the time-varying case as well. It turns out that the main workhorse in modern Darlington theory is the geometry of the so called Hankel map of the scattering transfer function to be embedded. This fact makes Darlington theory of considerably larger importance for the understanding of systems and their properties than the original synthesis question would seem to infer. Although the paper is entirely devoted to the theoretical question of existence of the Darlington embedding and its system theoretic implications, it does introduce the main algorithm used for practical Darlington synthesis, namely the 'square root algorithm' for external or inner-outer factorization, and discusses some of its implications in the final section.
\end{abstract}

\section{INTRODUCTION}

$\mathbf{T}$ RADITIONAL Darlington synthesis [1] is concerned with the realization of a rational and 'bounded real' transfer function $S(s)$ with bound 1 as a partial transfer operator of a 'lossless' transfer matrix $\Sigma(s)$, which then has the form

$$
\Sigma(s)=\left[\begin{array}{cc}
S(s) & \Sigma_{12}(s) \\
\Sigma_{21}(s) & \Sigma_{22}(s)
\end{array}\right]
$$

and in which the Smith-McMillan degree of $\Sigma$ is equal to that of $S$ (it is equal to the dimension of the state space of a minimal system theoretical realization for $S$ ). In addition to the embedding, a cascade realization of $\Sigma$, which can always be performed in the rational case, results in an attractive

Manuscript received May 1, 1998; revised August 1, 1998.

The author is with DIMES, Delft University of Technology, Delft, the Netherlands.

Publisher Item Identifier S 1057-7122(99)00540-1.

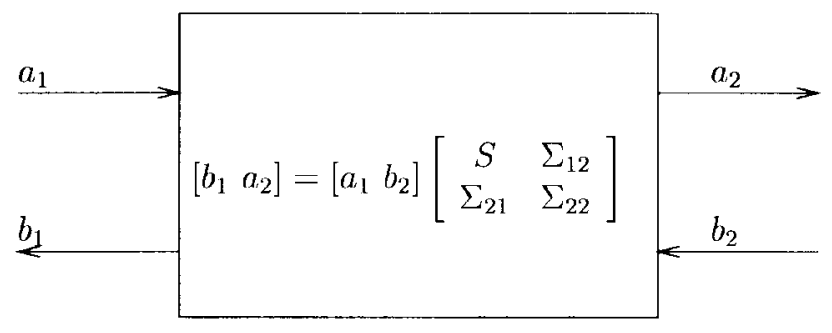

Fig. 1. The Darlington problem consists in the first place in embedding a contractive andcausal transfer function $S$ in a unitary and causal $\Sigma$. For a discussion of the meaning of the symbols used, see Section II.

realization of $S$ as well, especially from the point of view of selective behavior, i.e., bandpass and bandstop properties. Fig. 1 shows the Darlington set up. Signals on input and output wires carry the interpretation of incident and reflected waves in which the energy is measured as the norm squared quantity integrated over time.

In this paper we consider two important generalizations of the Darlington theory, one in the transform domain for systems with nonrational transfer functions and the other in the discrete time domain, for time-varying systems. We shall discover a striking parallel between these two cases, but some major and interesting differences as well. Generalizations of the Darlington theory to nonrational functions was a popular topic in the early seventies, and gave rise to contradictory results. For example, it has been stated that any contractive and causal transfer function $S(s)$ has a Darlington synthesis $\Sigma(s)$. If $\Sigma(s)$ is required to be unitary on the imaginary axis $s=j \omega$, the statement is certainly not true. Necessary and sufficient embedding conditions were discovered independently by the author [2] and D. S. Arov [3]. On the other hand, if the requirement is simply that $S(s)$ be embedded in an isometric $\Sigma(s)$ with a finite number of ports, then necessary and sufficient conditions are also known, and closely related to the classical Szegö condition for the existence of a 'spectral factorization', a particularly nice exposition of which is given in [4].

In recent times, the author and his collaborators have spent quite some effort in extending the Darlington theory to the discrete time, time-varying case, say a case that covers and extends classical linear algebra. It turns out that embedding anomalies which in the time-invariant case only occur for systems with infinite dimensional state space, already take place in time-varying systems with very low state dimensions. We shall report on these results further on in this paper. 
It turns out that the state-space formalism is the most effective vehicle to discuss Darlington synthesis. Darlington himself was working strictly with rational functions in the transform domain. Anderson and Vongpanitlerd [5] have demonstrated convincingly the power of the state space approach in their landmark book. More recent work on time-varying systems shows that Darlington theory does not need an analytical framework, it suffices with a purely algebraic theory. Darlington was working strictly with a scalar function $S(s)$, which he was embedding in a $2 \times 2$ matrix function of the variable $s$. Darlington's work was duly generalized by Belevitch [6], [7] and put in a practical context by Neirynck e.a. [8] leading to the effective and popular 'parametric methods' to synthesize lossless selective cascade filters. Subsequent to this work there were attempts to generalize it to multiple input multiple output systems ([7] and [9]) but these were not really successful, due to the lack of a good degree reduction criterion for multiport factorization. Such a criterion was first presented in [10] and lead to a new and effective method for multiport Darlington synthesis [11]. It turns out that the latter method actually generalizes to the nonrational case. We shall give a survey of these results later on.

For the sake of uniform treatment, and ease of relating to analytic properties, we shall put the theory in the $z$ domain, where the symbol $z$ can be interpreted as a causal shift operator (for mathematical ease we use $z$ instead of the " $z^{-1}$ " that is more common in the engineering literature), or just as $z=\frac{1-s}{1+s}$, where $s$ is the variable in the Laplace transform of continuous time functions. The theory remains in this way applicable to the ' $s$ '-domain through the bilinear transformation, whose inverse is $s=\frac{1-z}{1+z}$, and which maps the unit circle of the complex $z$-plane on the imaginary axis of the $s$-plane and the open unit disc of the $z$-plane onto the open right half plane of the $s$-plane (it is also the socalled Smith transform). The reason why it does not matter too much in which variable we work is precisely the algebraic nature of the Darlington theory, and the the fact that the bilinear transformation conserves the degree of polynomials after transformation.

\section{The BAsic DARlington Synthesis PROBlem}

We now work toward a general formulation of the basic Darlington synthesis problem in the $z$-plane context. There is some ambiguity in the literature concerning what is really 'Darlington synthesis', which we will explain further on. We start out considering an $m \times n$ transfer function $S(z)$ which can be thought of as representing a causal, discrete time system, although that interpretation could be a little strenuous when it concerns a continuous-time signal-but it does exist. If the system represented by $S(z)$ is stable in some sense (we already assumed it to be causal), then $S(z)$ will be analytic inside the unit disc and have some boundedness associated with it. There are several contexts appropriate for studying stability, we choose one which is natural to Darlington theory as it is concerned with propagation of energy, the Hilbert space context. The impulse response $s(t)$ corresponding to $S(z)$ defines bounded map of the convolution type between two spaces of time series whose energy is bounded. Such spaces are called 'of $\ell_{2}$ type'. As input space we take time series of the type $\left[\ldots, a_{-2}, a_{-1}, a_{0}, a_{1}, a_{2}, \ldots\right]$ where each $a_{k}$ belongs to a (possibly complex) vector space of the type $\mathbf{C}^{m}$, in which $\mathbf{C}$ indicates the complex numbers. (As a rule, we take vectors in the 'row convention', i.e. we write the multiplication of a vector $x$ with a matrix $A$ as $x A$. When we handle infinite series or infinite matrices, we single out the zero-th element by surrounding it with a box, for orientation purposes.) We write the Euclidean norm of a vector $a_{k}$ as $\left\|a_{k}\right\|_{2}$ and it equals

$$
\left\|a_{k}\right\|_{2}=\sqrt{\sum_{i=1}^{m}\left|a_{k}\right|_{i}^{2}}
$$

in which $\left[a_{k}\right]_{i}$ is the $i$-th (scalar) component of $a_{k}$. A time series $a=\left[a_{k}\right]_{k=-\infty}^{\infty}$ which is bounded in energy then has the norm

$$
\|a\|_{2}=\sqrt{\sum_{k=-\infty}^{\infty}\left\|a_{k}\right\|^{2}} .
$$

The space of such bounded time series is traditionally called $\ell_{2}^{m}$. A simple $m \times n$ matrix $A$ which maps a vector $a$ to a vector $b=a A$ will have an 'operator' norm given by

$$
\|A\|=\sup _{\|a\|_{2}=1}\left(\|a A\|_{2}\right)
$$

This norm is not a Euclidean norm on the $m * n$ space of entries of $A$, such a norm would be called a 'Frobenius norm':

$$
\|A\|_{F}=\sqrt{\sum_{i=1, k=1}^{(m, n)}\left|A_{i, k}\right|^{2}}
$$

A bounded input-bounded output (BIBO) transfer operator $\mathcal{S}$ between an input space $\ell_{2}^{m}$ and an output space $\ell_{2}^{n}$ will have the property that its Fourier transfer function $S\left(e^{j \theta}\right)$ is uniformly bounded on the open unit disc $\mathbf{D}=\{z:\|z\|<1\}$ of the complex plane in the sense that the collection of Euclidean matrix norms $\|S(z)\|$ has a finite upper bound when $\|z\|<1$. It is known in complex function theory (for a good textbook, see [12]) that such functions have radial limits $\lim _{\rho \rightarrow 1} S\left(\rho e^{j \theta}\right)$ to values $S\left(e^{j \theta}\right)$ on the unit circle $\mathbf{T}=\{z:|z|=1\}$ of the complex plane, whose norms are also uniformly bounded with the same bound. The space of such uniformly bounded matrix-functions on the unit disc is traditionally called the Hardy space $H_{\infty}^{m \times n}$. It is actually the subspace of measurable and uniformly bounded functions on the unit circle $L_{\infty}(\mathbf{T})$, which have a uniformly bounded analytic continuation to the open unit disc D. It turns out that the bound, which is denoted $\|S\|_{H_{\infty}}$ or equivalently $\|S\|_{L_{\infty}}$, is actually a least upper bound on the energy magnification between the input and the output of $\mathcal{S}$ [12], [13]. Hence, $\mathcal{S}$ will be BIBO stable for the energy norm on the input and output spaces iff $\|S\|_{H_{\infty}}<\infty$. If, 
in addition, the bound is actually equal or less than 1 , then the system with transfer function $S(z)$ will not amplify the input energy, it will have a passive input/output behavior, and should be realizable by a physically passive system. We say that in that case $\mathcal{S}$ is contractive (in the engineering literature, the term "bounded real' is also used, but we prefer the mathematical term because of the ambiguous connotation of the former). Such an $\mathcal{S}$ is characterized by the properties: ' $S(z)$ is analytic in $\{\|z\|<1\}$ and $\sup _{\|z\| \leq 1}\|S(z)\| \leq 1$ '. In particular we have that $S(z)$ is a causal transfer function and on the unit circle $\left\|S\left(e^{j \theta}\right)\right\| \leq 1$ (in which $j$ indicates the positive square root of -1 ). Note that the conditions ' $S(z)$ analytic in the open unit disc and $\left\|S\left(e^{j \theta}\right)\right\| \leq 1$ ' are not sufficient to guarantee that $S(z)$ is in $H_{\infty}$ (i.e. $\ell_{2}$-BIBO stable) - a counterexample is given by $S(z)=\exp \left(\frac{1+z}{1-z}\right)$, which is analytic but unbounded in $\mathrm{D}$, and of unit modulus a.e. on the unit circle.

We need a few additional notions from matrix or operator theory. The hermitian transform of a matrix $A$ is the matrix $A^{*}$ whose elements are defined by $\left[A^{*}\right]_{i j}=\bar{A}_{j i}$ in which the bar indicates complex conjugation. We say that $A$ is hermitian, if it is square and equals its hermitian transform, $A=A^{*}$. We say that $A$ is positive definite if it is hermitian and for all (row-)vectors $u$ of the right dimension we have $u A u^{*} \geq 0$. $A$ is strictly positive definite if $u A u^{*}=0 \Rightarrow u=0$. An $m \times n$ matrix $A$ actually defines a linear map from a (complex) $m$-dimensional vector space to a (complex) $n$-dimensional vector space through the assignment $u \mapsto y=u A$. We shall assume that on an $m$-dimensional vector space an inner product is defined as $(u, u)=u u^{*}$. The hermitian conjugate then defines what is called an adjoint map through the rule $\forall u, y:(u A, y)=\left(u, y A^{*}\right)\left(=u A y^{*}=u\left(y A^{*}\right)^{*}\right)$.

Since a transfer function also maps an input space, now of type $\ell_{2}^{m}$, to an output space, now of type $\ell_{2}^{n}$, we may extend the definitions to the natural in-product in $\ell_{2}$-type spaces. In the 'time domain' we have here an abstract map $\mathcal{S}: u \mapsto y=u \mathcal{S}$ given by convolution, while in the transfer domain the map is given by $z$-transform multiplication: $Y(z)=U(z) S(z)$. The relevant in-product in the input space (and likewise in the output space) is expressed in the time domain by

$$
\left(u_{1}, u_{2}\right)=\sum_{k=-\infty}^{\infty} u_{1 k} u_{2 k}^{*},
$$

and, via Parseval's theorem, in the transform domain by

$$
\left(u_{1}, u_{2}\right)=\int_{-\pi}^{\pi} U_{1}\left(e^{j \theta}\right)\left[U_{2}\left(e^{j \theta}\right)\right]^{*} \frac{d \theta}{2 \pi} .
$$

The adjoint of a transfer operator $\mathcal{S}$ will then be defined by the rule: $\forall u \forall y:(u \mathcal{S}, y)=\left(u, y \mathcal{S}^{*}\right)$. If $S(z)$ is the transfer function that corresponds to $\mathcal{S}$ and the $S\left(e^{j \theta}\right)$ are the matrix values that $S$ takes on the unit circle, then the transfer function corresponding to its adjoint takes the values $S_{*}\left(e^{j \theta}\right)=\left[S\left(e^{j \theta}\right)\right]^{*}$ on the unit circle. The corresponding transfer function (i.e. function of $z$ ) is then the so-called paraHermitian conjugate $S_{*}(z) \triangleq\left[S\left(\frac{1}{\bar{z}}\right)\right]^{*}$, which is anticausal when $S(z)$ is causal. It turns out that this transfer function defines an output-input map that produces an energy in-product related to the original by adjunction.
If $S(z)$ corresponds to a passive system, then it is contractive, i.e. $\|S\|_{H_{\infty}} \leq 1$. This will be the case iff $\forall z \in \mathbf{D}$ : $I-S(z)[S(z)]^{*} \geq 0$. (Notice that in general $[S(z)]^{*} \neq S_{*}(z)$ except on the unit circle $\left\{z: z=e^{j \theta}\right\}$-we must carefully distinguish the upper star, which indicates pointwise Hermitian conjugation, from the lower star, which denotes the analytical continuation of the hermitian conjugate on the unit circle.) We shall say that $S(z)$ is "isometric" if $\forall \theta: S\left(e^{j \theta}\right)\left[S\left(e^{j \theta}\right)\right]^{*}=I$ or, equivalently, $S(z) S_{*}(z) \equiv I$. In that case, the energy in the output $y=u \mathcal{S}$ is in all circumstances equal to the energy in the input $u$, and the system producing it must then be physically lossless. A transfer function characterization of this condition is obtained by analytic extension from the unit circle as $S(z) S_{*}(z)=I$ for whatever region in the complex plane wherein the extension is well-defined. We say that $S(z)$ is 'unitary' if, in addition to $S(z) S_{*}(z) \equiv I$, also $S_{*}(z) S(z) \equiv I$. This will be the case when $S(z)$ is isometric and square. If $S(z)$ is at the same time causal and unitary (i.e. it is in $H_{\infty}$ and it is unitary), then it is called inner in the mathematical literature, a term that we shall adopt. The term 'Darlington synthesis' of $S$ can then just as well be termed 'inner embedding' of $S$. Given a contractive, $m \times m S(z)$ it is not at all obvious that there exists an $m \times m \Sigma_{12}(z)$ such that the combined $\left[S(z) \Sigma_{12}(z)\right]$ is isometric. Nor is it obvious that $S(z)$ has an embedding into a unitary $\Sigma(z)$. In the next section we shall summarize the results known on these matters.

As the reader will have noticed, we avoid a physical terminology to indicate mathematical properties. The reason should be clear: there are many ways to describe a given physical situation mathematically. In our treatment, we use a 'scattering' framework throughout. This means that the signals carry energy in the form of a quadratic norm. That is, for example, the case when the input and output quantities of a circuit are incident or reflected waves. If, at a given port, the input quantity would be a voltage $v(t)$ and the output quantity a current $i(t)$, then the corresponding power transport would be measured by the real part of the mixed voltagecurrent product, $\Re\left(v(t) i(t)^{*}\right)$. Given a normalizing resistance value $r$ we can transform $v$ and $i$ to the wave quantities $a(t)=\frac{v(t)+r i(t)}{2 \sqrt{r}}, b(t)=\frac{v(t)-r i(t)}{2 \sqrt{r}}$ and the power transport becomes $\|a(t)\|^{2}-\|b(t)\|^{2}$, the difference between the power carried by an "incident wave" $a$ taken as input signal and the power carried by the "reflected wave" $b$ taken as output signal [14]. These power quantities are now strictly represented by quadratic norms (the energy then becomes the integral over time). It is known in filter theory that this is indeed a pertinent way of representing selective filters connected between lossy sources and loads [15].

Before proceeding to our main results, we need a few more notions from analytical function theory. Any (scalar) function $f(z)$ in $H_{\infty}$ can be factorized as $f(z)=\phi(z) f_{o}(z)$ in which $\phi(z)$ is inner (i.e. a causal transfer function of constant modulus 1 or pure phase function) and $f_{o}(z)$ which has the property of being outer. Although outerness has some nice analytical characterizations, which require some more theory than needed for this paper, we shall suffice here by stating that it is characterized by the property that it has an approximate 
causal inverse, i.e. that there exists a sequence of causal bounded functions $g_{i}(z)$ so that $\lim _{i \rightarrow \infty} f_{o}(z) g_{i}(z)=1$ in the quadratic sense on the unit circle. For all engineering purposes, $f_{o}(z)$ has a causal inverse, it is a "minimal phase function". The property extends to matrix functions: if $F(z) \in H_{\infty}^{m \times n}$, then $F(z)=U(z) F_{o}(z)$ in which $U(z)$ is inner, and $F_{o}(z)$ outer-see the discussion further on.

\section{ISOMETRIC AND UNITARY EMBEDDING-THE LTI CASE}

In the next three sections we give a number of results on isometric and unitary embedding for the LTI case. We start with some general considerations and then focus on systems with a finite dimensional state space, those are systems represented by a rational transfer function.

Suppose that an $m \times m$ causal and contractive transfer function $S(z)$ is given (we may assume $S$ to be square, if that would not be the case the addition of extra zero rows or columns would be adequate). Then the matrix function $W\left(e^{j \theta}\right)=I-S\left(e^{j \theta}\right) S\left(e^{j \theta}\right)^{*}$ is positive definite on the unit circle of the complex plane. We search for an $m \times m$ causal transfer function $\Sigma_{12}(z)$ such that $W\left(e^{j \theta}\right)=\Sigma_{12}\left(e^{j \theta}\right) \Sigma_{12}\left(e^{j \theta}\right)^{*}$-a spectral factorization of $I-S(z) S_{*}(z)=\Sigma_{12}(z) \Sigma_{12 *}(z)$. A first, fundamental, but not constructive, result was given by Masani and Wiener [16] - for a very nice treatment of the Masani Wiener theory, see Helson [4]. It states:

Theorem 1 (Masani-Wiener): Let the positive definite $m \times$ $m$ matrix function $W\left(e^{j \theta}\right)$ be invertible almost anywhere on the unit circle $\mathbf{T}$ of the complex plane. Then $W$ is factorable as $\Sigma_{12}\left(e^{j \theta}\right) \Sigma_{12}\left(e^{j \theta}\right) *$ if and only if

$$
\int_{-\pi}^{\pi} \log \operatorname{det} W\left(e^{j \theta}\right) \frac{d \theta}{2 \pi}>-\infty
$$

There is a (fairly straightforward) extension of the theorem to the case where $W$ is not invertible a.e., but here we stick to the generic case. The important point is that the factorization does not necessarily exist. The existence condition (2) for the matrix function case is an extension of the famous Szegö condition for the scalar case [17], and it states, loosely speaking, that the spectrum $W\left(e^{j \theta}\right)$ cannot be zero on more than a very thin set. For example, if $W\left(e^{j \theta}\right)$ has the behavior of an ideal low pass filter, then there would be an interval in which it is identically zero, and the condition would certainly be violated. The integral in (2) is known as the entropy of the process for which $W\left(e^{j \theta}\right)$ is the spectral density, and the property that it is larger than $-\infty$ corresponds to a stochastic property known as 'indeterminatedness' of the process. Be that as it may, if $S(z)$ is rational, then the Szegö condition will certainly be satisfied and the desired $\Sigma_{12}(z)$ will also exist. The point to be considered is the construction of $\Sigma_{12}(z)$ so that it has at most the same Smith-McMillan degree as $S(z)$. In the section on time-varying systems we shall given such a construction based on a state space representation for $S$, a construction, which is equally valid for the LTI case as a special case.

Of all the $\Sigma_{12}(z)$ of minimal degree, which satisfy $I-$ $S(z) S_{*}(z)=\Sigma_{12}(z) \Sigma_{12 *}(z)$, there is one of special interest, namely the one which is outer or minimal phase. This is the one that we would normally construct if we use the algorithms presented later (actually it does not matter which minimal one is chosen for the embedding construction to follow next). Let us assume that this is the $\Sigma_{12}$ selected, but at this point we do not yet assume rationality. Consider next the transfer function

$$
\left[S(z) \quad \Sigma_{12}(z)\right]
$$

which is isometric on $\mathbf{T}$. As the following endeavor, we wish to find causal transfer functions $\Sigma_{21}(z)$ and $\Sigma_{22}(z)$ so that the overall $2 m \times 2 m$ transfer function

$$
\Sigma(z)=\left[\begin{array}{cc}
S(z) & \Sigma_{12}(z) \\
\Sigma_{21}(z) & \Sigma_{22}(z)
\end{array}\right]
$$

is unitary on $\mathbf{T}$.

It has been claimed in the literature that this can always be done [18]. This is not true. One obvious property of $\Sigma(z)$ is that its elements have a special kind of analytic extension to outside the closed unit disc. Expressed in terms of the complex variable $z$, the unitarity of (3) says that

$$
\Sigma(z) \Sigma_{*}(z) \equiv I_{2 m}
$$

As an analytic function in the open unit disc $\mathrm{D}, \Sigma(z)$ is uniquely defined by its boundary values $\Sigma\left(e^{j \theta}\right)$, and it will have only discrete zeros in $\mathbf{D}$, as points $z \in \mathbf{D}$ where its rank drops from $2 m$ (this uniticity is a result of complex function analysis applied to $H_{\infty}$, see [12].) Since for $z \in \mathbf{T}$, $\Sigma_{*}(z)=\left[\Sigma\left(\frac{1}{z}\right)\right]^{*}$, and from (3) one sees that there will exist a continuation for $S(z)$ to the whole region outside the closed unit disc (the region $\mathbf{E}=\{z:|z|>1\}$ ) as $\left[\left[\Sigma\left(\frac{1}{\bar{z}}\right)\right]^{-1}\right]_{11}^{*}$, which certainly exists as a matrix of meromorphic entries in $\mathbf{E}$. We see that if there exists a unitary embedding for $S(z)$, then also the entries of $S(z)$ must have a meromorphic continuation to the complete region $\mathbf{E}$. Such $H_{\infty}$ functions are called pseudo-meromorphically continuable, and they form a class of transfer functions with special analyticity properties, namely the pseudo-meromorphic extension is unique and it has radial limits a.e. to the unit circle from points in E. Surely, rational transfer functions are such, but it is easy to cook up functions which do not satisfy the condition and necessarily will not be rational. An interesting example is $S(z)=(1 / \sqrt{z+3})$ which is obviously contractive in $\mathbf{D}$ (its maximum modulus in that region is $1 / \sqrt{2}$ ), it is analytically continuable everywhere across the unit circle, but not to the whole of $\mathbf{E}$, since the square root will have an essential singularity at the point $z=-3$ from which a branch cut will extend to $\infty$. It follows that this $S(z)$ has no unitary embedding, there is no Darlington synthesis for this $S$ !

The previous reasoning, convincing as it is, does not yet give a system or circuit theoretical clue as to why the embedding should not exist when the transfer function has no pseudomeromorphic continuation. We explore this point in the next section.

\section{REMEMBRANCE OF ThINGS PAST}

In [2], [19] the author showed that the existence of a Darlington synthesis for a contractive $m \times m$ scattering matrix $S(z)$ is closely related to a basic system theoretical property 
of $\mathcal{S}$. The property is of a geometric nature and carries over to situations where there is no transform available anymore, such as time-varying systems. In general terms (we shall make things more precise soon), the system's geometry viewed from an input-output point of view is characterized by four related spaces and a system induced map between them which is called the"Hankel map". Let $\mathbf{u}$ be the space of input signals and $\mathbf{y}$ the space of output signals (in our framework, both spaces will consist of vector time series which are bounded in energy, they are Hilbert spaces). Classical dynamic System Theory (for an original description see [20]) decomposes each space in a strict past and a future: $\mathbf{u}=\mathbf{u}_{p} \oplus \mathbf{u}_{f}(\oplus$ indicates decomposition in orthogonal subspaces- $\mathbf{u}$ and $\mathbf{y}$ are Hilbert spaces of the $\ell_{2}^{m}$-type). For example, with $u=$ $[\ldots,-2,-1,3,5,2, \ldots]$ we have $u_{p}=[\ldots,-2,-1,0,0, \ldots]$ and $u_{f}=[\ldots, 0,3,5,2, \ldots]$. Let $\mathbf{P}$ indicate the projection of a signal on his future part (i.e. $\mathbf{P}(u)=u_{f}$ ), then the Hankel map maps by definition $\mathbf{u}_{p}$ to $\mathbf{y}_{f}$ through the recipe:

$$
H_{S}: u_{p} \in \mathbf{u}_{p} \mapsto y_{f} \in \mathbf{y}_{f}: y_{f}=\mathbf{P}\left(u_{p} S\right) .
$$

It connects the (strict) past of the system to its future. This connection happens concretely through the state of the system, in the situation whereby nonzero input is only provided in the past (i.e. the input signal is zero from $t=0$ on): from its past, the system gleans information which it subsumes in its state, and then it uses this state to generate its future, under the assumption that future inputs are zero (in case the system is not linear a more sophisticated definition must be given, see [20], but we stick to the linear case here). Fig. 2 illustrates the situation. We can expect that the Hankel map yields important information on the structure of the system. Its kernel $\mathbf{k}$, consisting of the signals $\mathbf{k}=\left\{u_{p}: u_{p} H_{S}=0\right\}$ consists of all the (strict) past input signals whose information the system does not retain: its future response to such a signal is zero. The orthogonal complement to $\mathrm{k}$ in the past input space we call $\mathbf{h}=\mathbf{u}_{p} \ominus \mathbf{k}$, and it is a space characteristic for the information that the system does retain. It is also the range of $H_{S}^{*}$. We call it the natural input state space or reachability space of the system. At the output side there are the duals of these spaces. The range of the Hankel map, $\mathbf{h}_{o}=\operatorname{ran}\left(H_{S}\right)$ is the space of natural responses, those are the responses of the system when the input is kept equal to zero. It is a natural output state space or observability space for the system. Its orthogonal complement $\mathbf{k}_{o}=\mathbf{y}_{f} \ominus \mathbf{h}_{o}$ is actually the kernel of the adjoint map $H_{S}^{*}$, i.e. the set of outputs $\left\{y_{f}: y_{f} H_{S}^{*}=0\right\}$. All the spaces defined in this section have of course Fourier transforms, and their orthogonality relationships are preserved thanks to the Parseval theorem. We denote these Fourier transforms by a $(\cdot)^{\sim}$, e.g. $\mathbf{k}^{\sim}$ is the Fourier transform of $\mathbf{k}$ (for readers familiar with Fourier theory we mention that a space like $\ell_{2}^{m}([0, \infty))$ transforms to the celebrated Hardy space $H_{2}^{m}$ of the unit disc $\mathbf{T}$, so that $\mathbf{k}_{o}^{\sim}$ and $\mathbf{h}_{0}^{\sim}$ are subspaces of it. $\ell_{2}^{m}((-\infty,-1])$ then transforms to the orthogonal complement $\left(H_{2}^{m}\right)^{\perp}$ of $H_{2}^{m}$ in $L_{2}^{m}(\mathbf{T})$ ).

The nullspace $\mathbf{k}^{\sim}$ has the property that it is 'shift invariant' for the backward shift: $\mathbf{k}^{\sim} z^{-1} \subset \mathbf{k}^{\sim}$, i.e. if some input $U_{p}(z)$ in the strict past generates the zero state, then so does

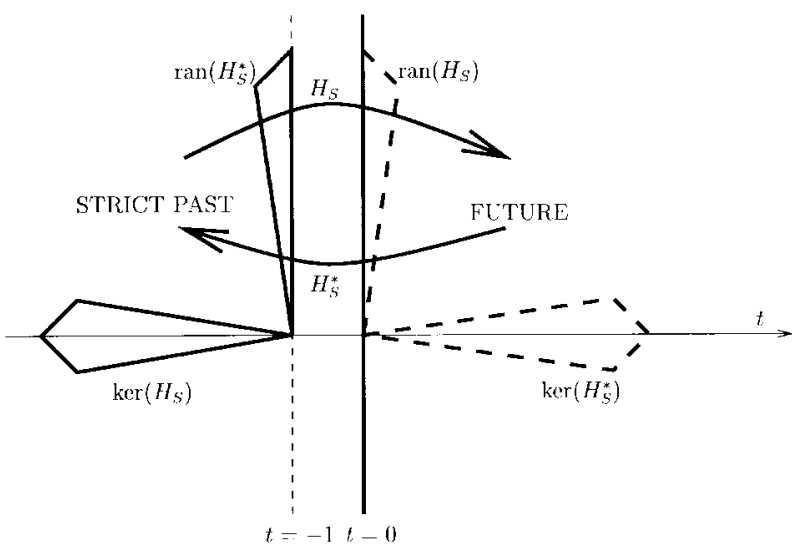

Fig. 2. The Hankel operator maps inputs in the strict past to outputs in the future.

$U_{p}(z) z^{-1}$. Such shift invariant spaces have remarkable mathematical properties described by the famous Beurling-Lax theorem [12], [4], [13] which we now introduce. It states that there exists a $k \times m$ transfer function $V_{\ell}(z)$ so that $V_{\ell *}(z)$ is isometric i.e. $\forall \theta:\left[V_{\ell}\left(e^{j \theta}\right)\right]^{*} V_{\ell}\left(e^{j \theta}\right]=I$ and all inputs in $\mathbf{k}^{\sim}$ are right multiples of $V_{\ell *}$ in the sense that $\mathbf{k}^{\sim}=F_{p}(z) V_{\ell *}$ for all $F_{p} \in \mathbf{f}_{p}^{\sim}$ with $\mathbf{f}_{p}$ an auxiliary $k$-dimensional space of the $\ell_{2}^{k}((-\infty,-1])$ type, (in the next section we shall meet rational examples which should make the present, general discussion less abstract). If we look at inputs in $\mathbf{k}^{\sim}$, then we see that for each point $e^{j \theta}$ of the unit circle, they form a $k$-dimensional subspace of the (pointwise) $m$-dimensional input space (in analytical function theory it is proven that the dimension $k$ is constant a.e.). Because $V_{\ell}$ is co-isometric, we necessarily have $k \leq m$. If $k<m$ then the nullspace is defective in dimension, and the corresponding input state space (the orthogonal complement of $\mathbf{k}$ ) will have to be very large, it cannot be a finite dimensional space since it must contain a space isomorphic to $\ell_{2}^{(m-k)}((-\infty,-1])$. For example, if $m=$ 1 , the scalar case, then $k$ is either 0 or 1 . In the first case, the nullspace $\mathbf{k}=\{0\}$ is trivial and the natural input state space $\mathbf{h}=\mathbf{u}$ becomes the entire input space, the system 'remembers' its complete input space, or, to put it differently, the state of the system is but a tampered version of the complete input. If, on the other hand, $k=m=1$, then $\mathbf{k}^{\sim}=\mathbf{u}_{p}^{\sim} e^{-j \theta} \phi\left(e^{j \theta}\right)^{*}$ in which $\phi\left(e^{j \theta}\right)$ is a scalar function of unit modulus. The natural input space becomes $\mathbf{h}^{\sim}=\mathbf{u}_{p}^{\sim} \ominus \mathbf{u}_{p}^{\sim} \phi_{*}$, which will be a finite dimensional space when $\phi$ is rational, and even otherwise it is still a very 'small' space, the system forgets almost everything from its past.

We say that a system is roomy [2], [19], iff $k=m$, i.e. iff the system has a pointwise fully dimensional nullspace. $V_{\ell}$ is in that case not only co-isometric, but unitary as well. Then we have that $V_{\ell *} S(z)=\Delta_{\ell *}$ in which $\Delta_{\ell}$ is a causal transfer function, and we obtain the left external factorization

$$
S(z)=V_{\ell}(z) \Delta_{\ell}^{*}(z)\left(=\left[V_{\ell *}(z)\right]^{-1} \Delta_{\ell *}\right)
$$

which displays the causal $S(z)$ as a ratio of two anticausal transfer functions. Hence $S(z)$, which is analytic in the open unit disc, has an extension (in the sense of radial pointwise limits) to a function which is meromorphic outside the unit 
disc, i.e. it is pseudo-meromorphically continuable (as stated before, such an extension is necessarily unique). The external factorization (7) is actually a "left coprime factorization" because it is minimal in the sense that every other such anticausal factorization will involve left multiples of $V_{\ell *}$ and $\Delta_{\ell *}$ (this fact needs some proof, see e.g. [19]). Hence, the coprime factorization involves automatically the existence of a pseudomeromorphic continuation for $S(z)$ as explained before. The converse is true as well: if $S(z)$ possesses a pseudomeromorphic continuation, then it also possesses a coprime factorization of the type (7). This fact can fairly easily be proven through the observation that if $S(z)$ is pseudomeromorphically continuable, then its nullspace $\mathbf{k}^{\sim}$ necessarily has full dimension $m$, since it will contain at least the space of all $m$-dimensional vectors with entries in the strict past that belong to the intersection of all the scalar input nullspaces of the entries $S_{i, k}(z)$.

A dual theory, now on the output nullspace $\mathbf{k}_{o}$, is of course also possible. In this case the Beurling-Lax theorem states that there exists an integer $k_{r}$ and a $k_{r} \times n$ causal and isometric function $V_{r}(z)$ such that $\mathbf{k}_{o}^{\sim}=\mathbf{g}_{f}^{\sim} V_{r}(z)$ for all $g_{f}$ belonging to an auxiliary input space $\mathbf{g}_{f}$ of the type $\ell_{2}^{k_{r}}([0, \infty))$. If the system is roomy then it will be meromorphically continuable, and it will follow that $k_{r}=n$. In that case we have that $V_{r}(z) S_{*}(z)=\Delta_{r}(z)$ for a causal $\Delta_{r}(z)$ and a unitary $V_{r}(z)$, and a right coprime external factorization

$$
S(z)=\Delta_{r *}(z) V_{r}(z)\left(=\Delta_{r *}(z)\left[V_{r *}(z)\right]^{-1}\right)
$$

follows, again exhibiting the pseudomeromorphic continuation. It is not too hard to prove that $\operatorname{det}\left(U_{\ell}(z)\right)=\operatorname{det}\left(U_{r}(z)\right)$, a quantity which can be used as a kind of 'generalized degree' [19].

\section{Generalized LTI DARLINGTON SyNTHESIS}

We are now ready to perform the general linear time invariant (LTI) Darlington synthesis in abstract terms. The method that we shall use can, however, easily be converted into a constructive or algorithmic technique, at least for the rational case, see the later sections of this paper. Let us assume that we are given an isometric and roomy $\Sigma_{1}(z)$. It can be obtained as $\left[S(z) \Sigma_{12}(z)\right]$, result of a spectral factorization, or else directly be given as an isometric $k \times m$ transfer function. Because of the roominess assumption, $\Sigma_{1}(z)$ will have a left coprime factorization:

$$
\Sigma_{1}(z)=V_{\ell}(z) \Delta_{\ell *}(z)
$$

in which $\Delta_{\ell *}(z)$ decomposes as $\left[\Delta_{\ell 1 *} \Delta_{\ell 2 *}\right]$ in accordance with $\left[S(z) \Sigma_{12}(z)\right]$. We see that $S(z)$ and $\Sigma_{12}(z)$ can share the same $V_{\ell}$ factor, it is in fact not too hard to prove that if $S(z)$ is roomy with left coprime unitary factor $V_{\ell}(z)$, then the minimal outer spectral factor $\Sigma_{12}(z)$ has an external factorization with left factor $V_{\ell}(z)$ as well. We formulate the generalized Darlington synthesis as a theorem.

Theorem 2: Let $\Sigma_{1}(z)$ be a causal and isometric transfer function. If $\Sigma_{1}(z)$ is roomy, then the right coprime factoriza- tion

$$
\Sigma_{1}(z)=\Delta_{r *} V_{r}(z)
$$

is such that $\Delta_{r * *}$ is constant and can be chosen $\left[\begin{array}{ll}I_{m} & 0\end{array}\right]$ and $V_{r}(z)$ is a (minimal) unitary embedding for $\Sigma_{1}(z)$ (and also for $S(z)$ which is part of $\Sigma_{1}(z)$ ). Conversely, suppose that $\Sigma_{1}(z)$ or $S(z)$ has a minimal unitary embedding, then it is roomy, and the Darlington embedding is actually a right external inner factor for $\Sigma_{1}(z)$.

Proof-Sketch: The proof makes use of a special property of coprime factorizations borrowed from standard Euclidean factorization theory and adapted to the $H_{\infty}$ case. In the standard theory we have that two polynomials $p_{1}(z)$ and $p_{2}(z)$ are coprime iff there exist polynomials $m(z)$ and $n(z)$ such that the Bezout equation $p_{1}(z) m(z)+p_{2}(z) n(z)=1$ is satisfied. The property extends to polynomial matrix functions, and with some effort also to our present case, where some added difficulties may occur because of the possible presence of zeros on the unit circle which actually do not participate in the division theory. The precise statement is [19]: two causal and bounded transfer functions $A(z)$ and $B(z)$ are left coprime (in the $H_{\infty}$ sense) iff there exist sequences $M_{i}(z)$ and $N_{i}(z)$ of causal and bounded transfer functions such that

$$
\lim _{i \rightarrow \infty}\left(A M_{i}+B N_{i}\right)=I
$$

for the entrywise quadratic norm on the unit circle.

(The difference with the standard case is that the $M_{i}$ and $N_{i}$ do not necessarily have a bounded limit themselves.) Two additional mathematical remarks are in order: 1) the limiting procedure given has as a consequence uniform convergence on compact subsets of the open unit disc and in particular pointwise convergence and 2) the property actually expresses the 'right outerness' of $[A(z) B(z)]$ ). If we apply this principle on the coprime right external factors for $\Sigma_{1}(z)=\Delta_{r *} V_{r}$ we deduce the existence of series of causal and bounded transfer matrices $M_{i}$ and $N_{i}$ which are such that

$$
\lim _{i \rightarrow \infty}\left(V_{r} M_{i}+\Delta_{r} N_{i}\right)=I
$$

Premultiplying with $\Delta_{r *}$ and using the fact that $\Delta_{r *} \Delta_{r}=I$ we find

$$
\Delta_{r *}=\lim _{i \rightarrow \infty}\left(\Sigma_{1} M_{i}+N_{i}\right)
$$

This expression asserts the existence of the limit in quadratic norm on the entries of $\Delta_{r *}$ from a series of elements which are all causal. Complex function theory then produces the result that $\Delta_{r *}$ must be causal as well. Since it is actually by definition anticausal, it can only be constant since this is the only type of transfer function that can be causal and anticausal at the same time. Hence, if the isometric $\Sigma_{1}(z)$ has an external factorization, which happens iff it is pseudomeromorphically continuable, then it will have a Darlington embedding. The converse was already evident from the discussion in the 
previous section in which we showed that an embedded $S(z)$ is necessarily pseudomeromorphically continuable.

A final remark for this section will provide the link with the time-varying theory to be treated furtheron. It seems that pseudomeromorphic continuability is the central property of transfer functions which allows for Darlington synthesis, but there is a time-domain criterion that is equivalent and more general. From the Darlington construction by external factorization given in Theorem 2 , it follows that $\Sigma_{1}(z)$ will have a Darlington synthesis iff its corresponding $\mathbf{k}_{o}^{\sim}$ has the full range property, i.e. its constituent functions span the space C $2 m \times 2 m$ at each point of the unit circle (a.e.). In the time domain, however, this property expresses an invariance which is of crucial importance, namely that $\mathbf{k}_{o}^{\sim}$ does not contain a subspace that is doubly invariant, i.e. for which $z \mathbf{k}_{O}^{\sim} \subset \mathbf{k}_{O}^{\sim}$ and $z^{-1} \mathbf{k}_{o}^{\sim} \subset \mathbf{k}_{o}^{\sim}$. It is this property that will determine the possibility for Darlington synthesis of time-varying systems for which there is no useful Laplace or $z$-transform.

\section{The TIME-VARYING CASE}

Now we consider a system $\mathbf{u} \mapsto \mathbf{y}$, which is time-varying, and given by a contractive transfer map $S$ (the distinction between $\mathcal{S}$ and $S(z)$ is not relevant anymore). The Darlington synthesis problem will consist in the solution of the question whether $S$ can be embedded in a causal unitary transfer function $\Sigma$,

$$
\Sigma=\left[\begin{array}{cc}
S & \Sigma_{12} \\
\Sigma_{21} & \Sigma_{22}
\end{array}\right]
$$

The input and output spaces are, as before, spaces of quadratically summable series, but because of the time variance we now have more freedom in allowing time variations from one time point to another. For example, we allow time varying dimensions for the vectors in the input and output spaces. We suppose that at a point $k$ in time the input vector $u_{k}$ belongs to a vector space $\mathbf{m}_{k}$, dependent on $k$, and likewise, $y_{k}$, belongs to a $\mathbf{n}_{k} \cdot \mathbf{m}_{k}$. has dimension $m_{k}$ while $\mathbf{n}_{k}$. has dimension $n_{k}$. The sequence of dimensions of the input sequence is then given by $\# \mathbf{m}=\left[\ldots, m_{-1}, m_{0}, m_{1}, \ldots\right]$. We assume that each input sequence considered is bounded in quadratic norm:

$$
\|u\|_{2}^{2}=\sum_{i=-\infty}^{\infty}\left\|u_{i}\right\|^{2}<\infty .
$$

We do allow that any number of spaces $\mathbf{m}_{k}$ is actually empty, i.e. has dimension zero. Such a space then simply corresponds to a 'place holder' [.] for which no value is available, i.e. at a certain time point there is no input data. In this way we can easily embed finite matrix theory in our framework: we take inputs as non existent up to $t=0$ and from some positive time point $t=N$ e.g. For higher precision of notation we shall henceforth denote the base input space as $\ell_{2}^{\mathbf{m}}$-in the sequel we shall have to extend it. The transfer operator $S$ maps an input $u$ to an output $y=u S$ and can hence be represented by

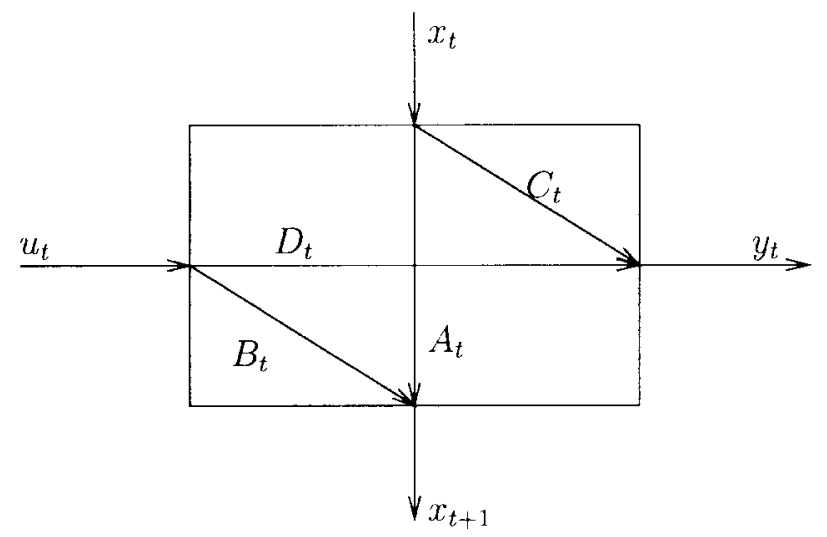

Fig. 3. The time-varying state space realization represents the calculation at at given point $t$ in the time sequence.

a (doubly infinite) matrix

$$
S=\left[\begin{array}{ccccc}
\ddots & \cdots & & & \\
& S_{-1,-1} & S_{-1,0} & S_{-1,1} & \\
\cdots & S_{0,-1} & S_{0,0} & S_{0,1} & \cdots \\
\cdots & S_{1,0} & S_{1,1} & \\
& & & \cdots & \ddots
\end{array}\right]
$$

in which each $S_{i, k}$ is a block matrix of dimensions $m_{i} \times n_{k}$. Causality is now expressed by a (block) upper triangular property: $S_{i, j}=0$ when $i>j$.

Connected to an upper transfer operator, there is a state space realization possible, based on the assumption that at each point in time $t$ the system uses a state $x_{t}$ that it has remembered from its past history, inputs a vector $u_{t}$, computes a new state $x_{(t+1)}$ and an output $y_{t}$, and then moves on to the next time point. Because of the linearity of the computation, the local state space representation takes the form

$$
\left[\begin{array}{ll}
x_{(t+1)} & y_{t}
\end{array}\right]=\left[\begin{array}{ll}
x_{t} & u_{t}
\end{array}\right]\left[\begin{array}{ll}
A_{t} & C_{t} \\
B_{t} & D_{t}
\end{array}\right]
$$

in which the 'realization' $\left\{A_{t}, B_{t}, C_{t}, D_{t}\right\}$ is a collection of linear operators (acting possibly on an infinite dimensional state space). The (time) series of states $\left[\ldots, x_{t}, \ldots\right]$ then belongs to a not yet defined series of spaces $\mathbf{b}=$ $\left[\ldots, \mathrm{b}_{t}, \ldots\right]$-see Fig. 3 .

On the various spaces of time sequences we can define standard operators as is also done in the time invariant case, but now we have to be a little more careful because of the changing dimensions, and the fact that operators which commute in the time invariant case may not commute anymore now. First there is the nondynamical operator of performing an instantaneous transformation on each entry of a time series: $\left[\ldots, u_{-1}, u_{0}, u_{1}, \ldots\right] \mapsto\left[\ldots, u_{-1} D_{-1}, u_{0} D_{0}, u_{1} D_{1}, \ldots\right]$ which corresponds to a diagonal operator

$$
D=\operatorname{diag}\left[\ldots, D_{-1}, D_{0}, D_{1}, \ldots\right] .
$$

$D$ will be bounded iff there is a uniform bound on its entries: $\|D\|=\sup _{t}\left\|D_{t}\right\|$, and it will be boundedly invertible iff each $D_{t}$ is square invertible, and there is a uniform bound on the collection of inverses $\sup _{t}\left\|D_{t}^{-1}\right\|<\infty$. Next there 
is the causal shift operator $Z:\left[\ldots, u_{-1}, u_{0}, u_{1}, \ldots\right] \mapsto$ $\left[\ldots, u_{-1}, u_{0}, u_{1}, \ldots\right] . Z^{-1}$ will then be the anticausal shift. If we collect the states of a computation in a series $x$, then we can express the state space realization in terms of the four diagonal operators $A=\operatorname{diag}\left[\ldots, A_{t}, \ldots\right] B=\operatorname{diag}\left[\ldots, B_{t}, \ldots\right]$, etc..., a

$$
\left\{\begin{array}{l}
x Z^{-1}=x A+u B \\
y=x C+u D
\end{array}\right.
$$

and we find, at least formally, the following expression for the corresponding transfer operator:

$$
S=D+B Z(I-A Z)^{-1} C
$$

the expression will make sense if a precise meaning to the operator $(I-A Z)^{-1}$ can be given (depending on the context, see further). An important remark at this point is that the causal shift $Z$ does not commute with the diagonal operators $Z A \neq A Z$ except when $A$ is Toeplitz, i.e. when all its entries on the main diagonal are equal (and then necessarily square of equal dimensions). Somewhat less is true, there is a kind of pseudocommutation, and we have to introduce a new notation for this:

$$
A Z=Z A^{(1)}
$$

in which $A^{(1)}$ is by definition a diagonal shift of the matrix $A$ in the South-East direction. We can apply further shifts, and, with some abuse of notation because the dimensions of the shift operator $Z$ vary along the main diagonal, we write $k$ applications of the diagonal shift as

$$
A^{(k)}=Z^{k} A Z^{-k} \text {. }
$$

$A^{(k)}$ is a diagonal operator equal to a version of $A$, shifted $k$ notches in the South-East direction. The causal operator $S$ can be represented by a collection of diagonal operators:

$$
S=\sum_{t=0}^{\infty} S_{k} Z^{k}
$$

in which $S_{k}=\operatorname{diag}\left[\ldots, S_{-1, k-1}, S_{0, k}, S_{1, k+1}, \ldots\right]$ It turns out that most of the time varying theory parallels the time invariant theory when one treats diagonals as ordinary 'scalars', respecting noncommutativities.

It is to be expected that the time-varying equivalent of the 'Hankel operator' will play a central role in a Darlington theory for time varying transfer operators. As we saw in the time invariant theory, the Hankel operator connects the 'strict past' of the system to its future, and therefore characterizes the structure of its state. However, a time-varying system may have a different state structure at each of its time points, so we have to define a Hankel operator at each point $t$. $H_{t}$ - the Hankel map at point $t$-will be defined by the map $\left[\ldots, u_{t-2}, u_{t-1}, 0,0, \ldots\right] \mapsto\left[y_{t}, y_{t+1}, y_{t+2}, \ldots\right]$ and it will be given by the matrix representation (dropping unneeded zero entries):

$$
H_{t}=\left[\begin{array}{cccc}
\vdots & \vdots & \vdots & \ddots \\
H_{t-2, t} & H_{t-2, t+1} & H_{t-2, t+2} & \cdots \\
H_{t-1, t} & H_{t-1, t+1} & H_{t-1, t+2} & \cdots
\end{array}\right] .
$$

The collection of the $\left\{H_{t}\right\}$ 's actually form the overall Hankel operator for the system $S$ under consideration. We build a system's map for which this collection is a natural operator. The trick is to consider not only one input time series at a given time point $t$, but to consider an appropriate sequence for each and every time point. Stacking these input (and the resulting output) time series as one input object produces the desired result. In this way, the input space becomes a space of objects of the type

$$
U=\left[\begin{array}{ccccccc}
\ddots & \vdots & \vdots & \vdots & \vdots & \vdots & . \\
\cdots & u_{-1,-2} & u_{-1,-1} & u_{-1,0} & u_{-1,1} & u_{-1,2} & \cdots \\
\cdots & u_{0,-2} & u_{0,-1} & u_{0,0} & u_{0,1} & u_{0,2} & \cdots \\
\cdots & u_{1,-2} & u_{1,-1} & u_{1,0} & u_{1,1} & u_{1,2} & \cdots \\
\cdots & u_{2,-2} & u_{2,-1} & u_{2,0} & u_{2,1} & u_{2,2} & \cdots \\
. & \vdots & \vdots & \vdots & \vdots & \vdots & \ddots
\end{array}\right] .
$$

In addition, we require that the complete scheme be bounded in energy, hence

$$
\|U\|_{2}^{2}=\sum_{i=-\infty, k=-\infty}^{\infty, \infty}\left\|u_{i, k}\right\|_{2}^{2}<\infty .
$$

The space so obtained we call $\mathcal{X}_{2}^{\mathcal{M}}$, it is again a (somewhat complicated) Hilbert space of quadratically summable time series. Since each row in this scheme corresponds to a simple time series for time point $t$, we have that $U$ can be decomposed in a strict past $U_{p}$ and a strict future $U_{f}$ so that $\left[U_{p}\right]_{i, k}=u_{i, k}$ when $i>k$, and otherwise zero, while $\left[U_{f}\right]_{i, k}=u_{i, k}$ when $i \leq k$ and otherwise zero. The space of the $U_{f}$ (i.e. the 'future') we shall now call $\mathcal{U}_{2}$, while the space of the $U_{p}$ (i.e. the 'strict past') we shall call $\mathcal{L}_{2} Z^{-1}$. We shall denote the projection of $\mathcal{X}_{2}$ on $\mathcal{U}_{2}$ by $\mathrm{P}$ and the projection on the orthogonal complement $\mathcal{L}_{2} Z^{-1}, \mathbf{P}^{\prime}$ (these are genuine orthogonal projections on the extended input and output spaces and $\mathbf{P}^{\prime}+\mathbf{P}=I$ ).

The action of a causal operator $S$ on such a scheme is easy to tally, we just have to stack inputs and outputs:

$$
\begin{aligned}
& {\left[\begin{array}{cccccc}
\cdots & u_{-1,-1} & u_{-1,0} & u_{-1,1} & u_{-1,2} & \cdots \\
\cdots & u_{0,-1} & u_{0,0} & u_{0,1} & u_{0,2} & \cdots \\
\cdots & u_{1,-1} & u_{1,0} & u_{1,1} & u_{1,2} & \cdots
\end{array}\right]} \\
& \times\left[\begin{array}{cccccc}
\ddots & \vdots & \vdots & \vdots & \vdots & \cdot \\
& S_{-1,-1} & S_{-1,0} & S_{-1,1} & S_{-1,2} & \cdots \\
& & S_{0,0} & S_{0,1} & S_{0,2} & \cdots \\
& & & S_{1,1} & S_{1,2} & \cdots \\
& & & & \ddots & \cdots \\
& & & & & \ddots
\end{array}\right] \\
& =\left[\begin{array}{cccccc}
\cdots & y_{-1,-1} & y_{-1,0} & y-1,1 & y_{-1,2} & \cdots \\
\cdots & y_{0,-1} & y_{0,0} & y_{0,1} & y_{0,2} & \cdots \\
\cdots & y_{1,-1} & y_{1,0} & y_{1,1} & y_{1,2} & \cdots
\end{array}\right]
\end{aligned}
$$




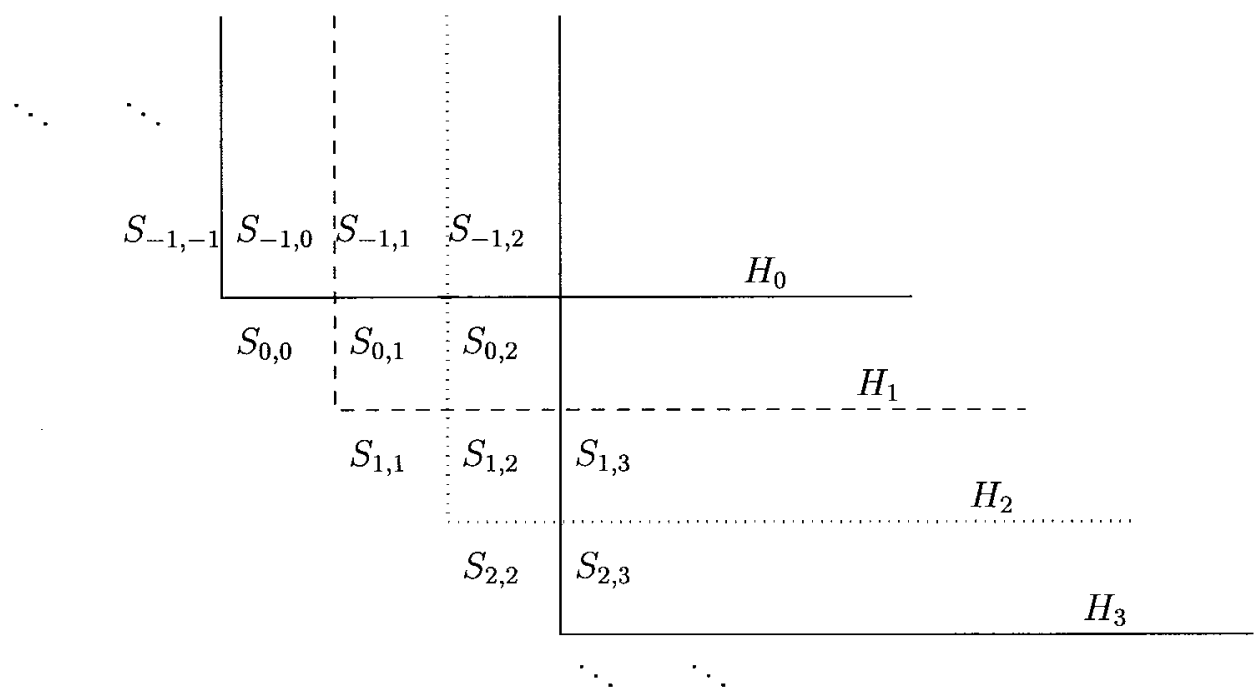

Fig. 4. The time-varying Hankel operator consists in a collection of matrices each of which maps an input in the strict past to an output in the future relative to a point $t$.

We are now able to define the global Hankel operator for $S$ as

$$
H_{S}=\left.\mathbf{P}(\cdot S)\right|_{\mathcal{L}_{2} Z^{-1}}\left(=\cdot \mathbf{P}^{\prime} S \mathbf{P}\right) .
$$

It consists in applying $S$ to elements of $\mathcal{X}_{2}$ which are restricted to the strict past, and looking at the effect only from time $t$ on, for each $t$, in one global operator. Since $H_{S}$ maps a two dimensional scheme to another two dimensional scheme, it is actually a tensor. At each time point $t, H_{S}$ specializes to a 'snapshot' which is obtained by looking at its effect on the $t$-th row of the input and looking at how it produces the $t$ th row of the output-this turns out to be the local Hankel matrix $H_{t}$. A graphical representation of the Hankel operator is shown in Fig. 4.

Just as in the LTI case, the Hankel operator will generate the system's state geometry. The following spaces are important:

- Natural Input State Space $\mathcal{H}=\operatorname{ran}\left(H_{S}^{*}\right)$

- Natural Output State Space $\mathcal{H}_{o}=\operatorname{ran}\left(H_{S}\right)$

- Input Nullspace $\mathcal{K}=\operatorname{ker}\left(H_{S}\right)$

- Output Nullspace $\mathcal{K}_{o}=\operatorname{ker}\left(H_{S}^{*}\right)$

The crucial property of these nullspaces are their invariances. We see that $\mathcal{K}$ is invariant for the left application of a diagonal $D$ and of the anticausal shift $Z^{-1}$ :

$$
\left\{\begin{array}{l}
D \mathcal{K} \subset \mathcal{K} \\
Z^{-1} \mathcal{K} \subset \mathcal{K}
\end{array}\right.
$$

Dually, $\mathcal{K}_{o}$ is invariant for the application of a diagonal $D$ and the causal shift:

$$
\left\{\begin{array}{lll}
D \mathcal{K}_{o} & \subset & \mathcal{K}_{o} \\
Z \mathcal{K}_{o} & \subset & \mathcal{K}_{o}
\end{array}\right.
$$

We call a $D$-invariant space a sliced space, because if $U$ belongs to it, then any row of $U$ with all other rows put to zero also belongs to it. A sliced space has a basis which consists of time slices: for each time index $t$ there is a basis for the corresponding row. A generalized Beurling-Lax theorem in this setting is as follows:
Theorem 3 (Generalized Beurling-Lax): Any left DZinvariant subspace $\mathcal{K}_{o}$ of $\mathcal{U}_{2}^{\mathcal{N}}$ has the form

$$
\mathcal{K}_{o}=\mathcal{U}_{2}^{\mathcal{K}} V
$$

for some causal and isometric operator $V$ (i.e. $V V^{*}=I$ ).

Sketch of Proof: One considers the (so called wandering) subspace $\mathcal{R}=\mathcal{K}_{o} \ominus Z \mathcal{K}_{o}$ and realizes that it is a sliced space as well. An orthonormal sliced basis for $\mathcal{R}$ produces the operator $V$ as the collection of these base vectors itself. This construction is identical to the construction used in the classical case. For details see [21].

The (generalized) Beurling-Lax theorem leads, just as in the classical case, to the construction of external factorizations for the operator $S$ and to Darlington synthesis, and will provide the conditions under which it is possible. The time-varying case is considerably more involved than the LTI case, and although we are able to formulate in abstract form necessary and sufficient conditions for the Darlington embedding to exist (Theorem 6), to treat the embedding concretely let us assume at this point that we dispose of a concrete stable state space description for $S$, i.e. we assume that (1) $S$ is locally finite, that is, it has a realization based on a collection $\mathcal{B}_{k}$ of finite dimensional local state spaces for each time point $k$ and which is given, as explained above, by diagonal operators $\{A, B, C, D\}$ consisting of diagonals of finite matrices and (2) the realization is such that the spectral radius $\sigma(A Z)$ of the operator $A Z$ is strictly less than one. The spectral radius is defined as

$$
\sigma(A Z)=\lim _{k \rightarrow \infty}\left\|(A Z)^{k}\right\|^{\frac{1}{k}} .
$$

We denote this positive number as $\ell_{A}=\sigma(A Z)$. It is actually equal to

$$
\ell_{A}=\lim _{k \rightarrow \infty}\left\|A \cdot A^{(-1)} \cdots A^{(-k+1)}\right\|^{\frac{1}{k}}
$$

which follows from working out the power product $(A Z)^{k}$. When $\ell_{A}<1$ we say that the system has a uniformly 
exponentially stable or ue-stable realization. When $\ell_{A}<1$ then left and right external factorizations for $S$ are easy to produce. They are based on the construction of so called normal forms for the realization $\{A, B, C, D\}$. We say that the realization is in output normal form if $[A C]$ is isometric, i.e. if $A A^{*}+C C^{*}=I$, i.e. for all $k, A_{k} A_{k}^{*}+C_{k} C_{k}^{*}=1$. Dually, it will be in input normal form, if $\left[A^{*} B^{*}\right]$ is isometric, i.e. $A^{*} A+B^{*} B=I$. Given a ue-stable realization, both the output and input normal forms can easily be constructed through a state transformation. Such a state transformation takes the character of a bounded and boundedly invertible diagonal map $R$ which transforms the state $x$ to $x^{\prime}=x R$ so that for each $k, x_{k}^{\prime}=x_{k} R_{k}$. If one applies this transformation on the state, then one obtains a new, equivalent realization:

$$
\left\{\begin{array}{l}
x_{k+1}^{\prime}=x_{k}^{\prime} R_{k}^{-1} A_{k} R_{k+1}+u_{k} B_{k} R_{k+1} \\
y_{k}=x_{k}^{\prime} R_{k}^{-1} C_{k}+u_{k} D_{k}
\end{array}\right.
$$

in other words, the realization $\{A, B, C, D\}$ transforms to the new equivalent realization $\left\{R^{-1} A R^{(-1)}, B R^{(-1)}, R^{-1} C, D\right\}$ (in which $\cdot(-1)$ represents the diagonal shift upward in the North-West direction, of course). To obtain, say, the output normal form, we must find $R$ so that $\left[\begin{array}{ll}R^{-1} A R^{(-1)} & R^{-1} C\end{array}\right]$ is isometric. Writing $G_{O}=R R^{*}$, this amounts to solving the equation:

$$
A G_{o}^{(-1)} A^{*}+C C^{*}=G_{o}
$$

or, in component form,

$$
A_{k} G_{o(k+1)} A_{k}^{*}+C_{k} C_{k}^{*}=G_{o k} .
$$

These recursive equations are called 'Lyapunov-Stein' equations. When $A$ is ue-stable, then they have a unique solution, which with, for $i=1,2, \ldots$,

$$
A^{\{i\}}=A A^{(-1)} \cdots A^{(-i+1)}
$$

and $A^{\{0\}}=I$, is found by solving (23) as a fixed point equation:

$$
G_{o}=\sum_{i=0}^{\infty} A^{\{i\}} C^{(-i)}\left[A^{\{i\}} C^{(-i)}\right]^{*} .
$$

If we define $\mathcal{O}=\left[\begin{array}{llll}C & A C^{(-1)} & A A^{(-1)} C^{(-2)} & \cdots\end{array}\right]$ as the observability operator, then we see that $G_{O}$ is actually a generalized Gramian $G_{0}=\mathcal{O O}^{*}$. To find the $R$ needed for the output normal form, we next have to factor $G_{0}=R R^{*}$, which of course should be possible, since all the individual matrices $G_{o k}$ are positive semi-definite and can be factored as $G_{o k}=R_{k} R_{k}^{*}$.

So far so good, but to achieve the output normal form, we need the bounded invertibility of $R$. This means that the individual $R_{k}$ must be invertible, and that their inverses must have a uniform upper bound. Alternatively, $G_{0}$ must have a definite lower bound, i.e. there must exist an $\epsilon>0$ such that $G_{0}>\epsilon I$. In other words, the system must be what we call uniformly observable. It should be clear that this is not always the case, and we obtain the result: a locally finite system which is ue-stable will have a ue-stable output normal form if it is uniformly observable. (There is a somewhat more involved converse to this property, which we skip for technical reasons.)
When a ue-stable system is uniformly observable, then it will have a right external factorization. The construction of the factorization is based on the output normal form. We saw in the preceeding paragraph that the system will have such a ue-stable form if it is uniformly observable. Suppose now that $\{A, B, C, D\}$ is in output normal form, that is $A A^{*}+C C^{*}=$ $I$. This means that each $\left[A_{k} C_{k}\right]$ is actually isometric. Let us now define two new diagonal operators $\left[\begin{array}{ll}B_{r} & D_{r}\end{array}\right]$ so that the realization

$$
\left[\begin{array}{cc}
A & C \\
B_{r} & D_{r}
\end{array}\right]
$$

is unitary, and let $U_{r}$ be the corresponding operator,

$$
U_{r}=D_{r}+B_{r} Z(I-A Z)^{-1} C .
$$

To assess the properties of $U_{r}$ we need the following lemma, which plays a central role in the subsequent discussion on Darlington realizations:

Lemma 1: Suppose that $U=D+B Z(I-A Z)^{-1} C$ is a transfer operator which is ue-stable and for which the realization operator

$$
\left[\begin{array}{ll}
A & C \\
B & D
\end{array}\right]
$$

is unitary. Then $U$ is unitary as well, i.e. $U U^{*}=I$ and $U^{*} U=I$

Sketch of Proof: The lemma can fairly easily be checked by direct calculation, the crucial point in the verification is the existence and boundedness of the inverse $(I-A Z)^{-1}$, which is assured by the ue-stable assumption.

Hence $U_{r}$, as defined, will be unitary if $A$ is ue-stable. It turns out to be the right inner factor for the right external, coprime factorization of $S$. This we see simply by computing $\Delta_{r}=U_{r} S^{*}$. During the computation one needs the (generalized) partial fraction decomposition of the product $Z(I-A Z)^{-1} C C^{*}(I-A Z)^{-*} Z^{*}$, which, because of $A A^{*}+$ $C C^{*}=I$ is easily seen to equal $I+Z(I-A Z)^{-1} A+A^{*}(I-$ $A Z)^{-*} Z^{*}$. Hence (we write ' $X^{-*}$ ' for $\left(X^{-1}\right)^{*}=\left(X^{*}\right)^{-1}$ )

$$
\begin{aligned}
\Delta_{r}= & B_{r} B^{*}+D_{r} D^{*}+B_{r} Z(I-A Z)^{-1}\left[A B^{*}+C D^{*}\right] \\
& +\left[B_{r} A^{*}+D_{r} C^{*}\right](I-A Z)^{-*} Z^{*} B^{*} .
\end{aligned}
$$

The last term in this expression is zero, because of the unitarity of the realization for $U_{r}$. Hence, a realization for $\Delta_{r}$ is given by

$$
\boldsymbol{\Delta}_{\mathbf{r}}=\left[\begin{array}{cc}
A & A B^{*}+C D^{*} \\
B_{r} & B_{r} B^{*}+D_{r} D^{*}
\end{array}\right] .
$$

Together these relations produce

$$
\left[\begin{array}{cc}
A & C \\
B_{r} & D_{r}
\end{array}\right]\left[\begin{array}{cc}
A^{*} & B^{*} \\
C^{*} & D^{*}
\end{array}\right]=\left[\begin{array}{cc}
I & C_{\Delta_{r}} \\
0 & D_{\Delta_{r}}
\end{array}\right]
$$

or, if the original realization $\mathbf{S}$ for $S$ would not have been in output normal form (and further explicited per time point):

$$
\left[\begin{array}{cc}
A_{r k} & C_{r k} \\
B_{r k} & C_{r k}
\end{array}\right]\left[\begin{array}{ll}
R_{k+1}^{*} A_{k}^{*} & B_{k}^{*} \\
R_{k+1}^{*} C_{k}^{*} & D_{k}^{*}
\end{array}\right]=\left[\begin{array}{cc}
R_{k}^{*} & C_{\Delta_{r k}} \\
0 & D_{\Delta_{r k}}
\end{array}\right]
$$

in which now $A_{r k}=R_{k}^{-1} A_{k} R_{k}^{(-1)}$ and $C_{r k}=R_{k}^{-1} C_{k}$. At each time point $k$, this equation has the form of the famous 
Jacobi $Q-R$ factorization of numerical linear algebra. If we assume that at time point $k$ we know $R_{k+1}$ from the previous calculation, then the matrix

$$
\left[\begin{array}{ll}
R_{k+1}^{*} A_{k}^{*} & B_{k}^{*} \\
R_{k+1}^{*} C_{k}^{*} & D_{k}^{*}
\end{array}\right]
$$

is known, and is transformed by the unitary ( $Q$-type) matrix

$$
\left[\begin{array}{cc}
A_{r k} & C_{r k} \\
B_{r k} & D_{r k}
\end{array}\right]
$$

to upper-triangular form (at least to block-upper form, but nothing prevents a further transformation to fully upper). The transformation produces $R_{k}$ which can then be used in the calculation at the next time point $k-1$, etc... recursively.

We have obtained the following result:

Theorem 4: Suppose that $S$ is a ue-stable, locally finite transfer function which is uniformly observable, then it has a right coprime external factorization given by

$$
S=\Delta_{r}^{*} U_{r}
$$

in which $U_{r}$ is inner. $\Delta_{r}$ and $U_{r}$ can be found by the square root algorithm of (27).

A kind of converse of the theorem is fairly easily demonstrated by construction of 'counterexamples', namely by constructing an $S$ with a ue-stable realization which is not uniformly observable. Such an $S$ will normally not have an external factorization. The author does not know whether the case where $S$ has a ue-stable realization which is not uniformly observable and for which a Darlington embedding exists actually do exist (he thinks they do), but normally the singularity of the Gramian will give rise to the existence of what we shall call a defect space, and we shall see in Theorem 5 that then a Darlington embedding does not exist.

Of course, there is a dual to the previous theorem, based on the input normal form. For that we need the notion of 'uniform reachability' which will involve the reachability operator $\mathcal{R}$ and the reachability Gramian $G_{r}=\mathcal{R}^{*} \mathcal{R}$. We shall say that the realization is uniformly reachable if there exists an $\epsilon$ such that $G_{r}>\epsilon I$. We obtain:

Theorem 5: Suppose that $S$ is a ue-stable, locally finite transfer function which is uniformly reachable, then it has a left coprime factorization given by

$$
S=U_{l} \Delta_{l}^{*}
$$

in which $U_{l}$ is inner. $\Delta_{l}$ and $U_{l}$ can be found by an appropriate square root algorithm.

In contrast to what happens in the LTI case, it is possible that a ue-stable time-varying system possesses a left external factorization but not a right one and vice versa. This will be the case, in general, when the system has a uniformly reachable ue-realization which is not uniformly observable or vice-versa, and this can already easily happen with systems that are locally finite. In the time invariant case all systems with finite dimensional state spaces have right and left coprime factorizations. In contrast to the claim that 'time-varying system theory is but a small extension of time invariant theory' we see that the two types of systems have very different properties indeed!
We terminate this section by showing how Darlington synthesis works for isometric systems, the more general (contractive) case is treated in the next section. Suppose that $S$ is isometric, i.e. $S S^{*}=I$ and that it is locally finite, i.e. that it has a finite dimensional state space realization at each time point. We proceed by constructing a particular realization $\mathbf{S}$ for $S$, which is itself isometric, and by trying to embed it in a larger realization which is unitary and hence a candidate for an inner transfer function $\Sigma$ that embeds $S$ as

$$
\Sigma=\left[\begin{array}{c}
S \\
\Sigma_{2}
\end{array}\right]
$$

As we saw before in the LTI case, the properties of the output state space $\mathcal{H}_{o}$ of $S$ will be crucial to the Darlington embedding theory and we may encounter some possibly very strange behavior here. An important property of an isometric system like $S$ is the fact that its output nullspace $\mathcal{K}_{o}$ actually contains the space $\mathcal{U}_{2}^{\mathcal{M}} S$ (this is the space of outputs to causal inputs under the action of the isometric operator $S$ ). Hence, $\mathcal{U}_{2}^{\mathcal{N}} \supset \mathcal{H}_{o}(S) \oplus \mathcal{U}_{2}^{\mathcal{M}} S$. But $\mathcal{K}_{o}$ may be larger. It will also contain the $\left.\operatorname{kernel} \operatorname{ker}\left(\cdot S^{*}\right)\right|_{\mathcal{U}_{2}^{\mathcal{M}}}$, by definition, and clearly $\left.\operatorname{ker}\left(\cdot S^{*}\right)\right|_{\mathcal{U}_{2}^{\mathcal{M}}} \perp \mathcal{U}_{2}^{\mathcal{N}} S \oplus \mathcal{H}_{o}(S)$. The 'algebraic' (i.e. unqualified) nullspace $\operatorname{ker}\left(\cdot S^{*}\right)$ of $S^{*}$ will not only contain $\left.\operatorname{ker}\left(\cdot S^{*}\right)\right|_{\mathcal{U}_{2}^{\mathcal{N}}}$, which is a left $Z$-invariant space, but also the latter's left shifts $\forall k \geq 1:\left.Z^{-k} \operatorname{ker}\left(\cdot S^{*}\right)\right|_{\mathcal{U}_{2}^{\mathcal{N}}}$, and hence their closed union, which we denote as

$$
\mathcal{K}_{o}^{\prime}=\left.\bigvee_{k=0}^{\infty} Z^{-k} \operatorname{ker}\left(\cdot S^{*}\right)\right|_{\mathcal{U}_{2}^{N}} .
$$

The space $\left.\operatorname{ker}\left(\cdot S^{*}\right)\right|_{\mathcal{U}_{2}^{\mathcal{N}}}$ turns out to be the characteristic output nullspace of the complementary entry $\Sigma_{2},\left.\operatorname{ker}\left(\cdot S^{*}\right)\right|_{\mathcal{U}_{2}^{N}}=$ $\mathcal{U}_{2}^{\mathcal{K}} \Sigma_{2}$ for an appropriate sequence of input spaces $\mathcal{K}$ complementary to $\mathcal{M}$. There is more the matter, however. The 'algebraic nullspace' $\operatorname{ker}\left(\cdot S^{*}\right)$ may be larger than $\mathcal{K}_{O}^{\prime}$. We denote that remaining space as

$$
\mathcal{K}_{o}^{\prime \prime}=\operatorname{ker}\left(\cdot S^{*}\right) \ominus \mathcal{K}_{o}^{\prime}
$$

and will call it the right defect space of $S$. The possible existence of this space has sometimes been overlooked in the literature, but its properties appear to be crucial to the existence of the Darlington embedding.

The following theorem (which does not assume local finiteness) gives, in addition to the essential properties needed, the Darlington theory for isometric systems in abstract form.

Theorem 6: Let $S$ be a causal isometric transfer operator and let $\mathcal{H}_{o}(S)$ be its natural output state space and $\mathcal{K}_{o}^{\prime \prime}(S)$ its right defect space. The output space $\mathcal{U}_{2}^{\mathcal{N}}$ then decomposes as

$$
\mathcal{U}_{2}^{\mathcal{N}}=\left.\mathcal{H}_{o}(S) \oplus \mathcal{U}_{2}^{\mathcal{M}} S \oplus \operatorname{ker}\left(\cdot S^{*}\right)\right|_{\mathcal{U}_{2}^{\mathcal{N}}}
$$

There exists an isometric operator $\Sigma_{2}$ with the same output state space $\mathcal{H}_{O}(S)$ and defect space $\mathcal{K}_{o}^{\prime \prime}(S)$ and such that, for some input space sequence $\mathcal{K}$,

$$
\left.\operatorname{ker}\left(\cdot S^{*}\right)\right|_{\mathcal{U}_{2}^{N}}=\mathcal{U}_{2}^{\mathcal{K}} \Sigma_{2}
$$

The operator

$$
\Sigma=\left[\begin{array}{c}
S \\
\Sigma_{2}
\end{array}\right]
$$


will then be isometric as well, and such that $\left.\operatorname{ker}\left(\cdot \Sigma^{*}\right)\right|_{\mathcal{U}_{2}^{\mathcal{N}}}=$ $\{0\} . \Sigma$ will be unitary (hence inner) iff the defect space $\mathcal{K}_{o}^{\prime \prime}=\{0\}$.

Sketch of Proof: The theorem is a direct consequence of the generalized Beurling-Lax representation theorem applied to the left DZ-invariant subspace $\left.\operatorname{ker}\left(\cdot S^{*}\right)\right|_{\mathcal{U}_{2}^{\mathcal{N}}} \subset \mathcal{U}_{2}^{\mathcal{M}}$ : $\left.\operatorname{ker}\left(\cdot S^{*}\right)\right|_{\left.\mathcal{U}_{2}^{N}\right)}=\mathcal{U}_{2}^{\mathcal{K}} \Sigma_{2}$. Furthermore, it is known from functional analysis that an isometric operator will actually be unitary iff it has full range, in the present case iff $\mathcal{K}_{\circ}^{\prime \prime}=\{0\}$. For a complete, formal proof we refer to the book [21].

In the case of ues locally finite systems, the theorem leads to the following construction for the embedding. First, an isometric realization for $S$ can be obtained from an orthonormal basis for the natural output state space $\mathcal{H}_{o}(S)$. Let, at time point $k, \mathbf{F}_{o k}$ be an orthonormal basis for the range of the Hankel matrix $H_{k}$, which is assumed to be of finite dimension $\delta_{k}$. Assembling all these bases as slices of an overall operator $\mathbf{F}_{o}$, we see that $\mathbf{F}_{o}$ has the form of an upper operator. The next step is the assertion that there exist diagonal operators $A$ and $C$ so that $\mathbf{F}_{o}$ can be represented as

$$
\mathbf{F}_{o}=C+A Z \mathbf{F}_{o}
$$

This fact is basic to state space realization theory and follows directly from the shift-invariance property of $\mathcal{H}_{o}(S)$ which states that $\mathbf{P}\left(Z^{*} \mathcal{H}_{o}(S)\right) \subset \mathcal{H}_{o}(S)$. Hence, given the choice of basis, we may recover corresponding diagonal operators $C$ and $A$ by the recipe: $C=\mathbf{P}_{0}\left(\mathbf{F}_{o}\right)$ and $A^{(-1)}=\mathbf{P}_{o}\left(Z^{-1} \mathbf{F}_{o} \mathbf{F}_{o}^{*}\right)$, in which we have used the orthonormality $\mathbf{P}_{0}\left(\mathbf{F}_{o} \mathbf{F}_{o}^{*}\right)=I$. The corresponding $[A C]$ will automatically be isometric, but $A$ is not necessarily ue-stable. An isometric realization for $S$ is next found by determining $B$ and $D$ from the relation $S=D+$ $B Z \mathbf{F}_{o}$, specifically, $D=\mathbf{P}_{0}(S), B^{(-1)}=\mathbf{P}_{o}\left(Z^{-1} S \mathbf{F}_{o}^{*}\right)$. The point is that, in contrast to traditional realization theory, we may not be able to express $S$ directly as $D+B Z(I-A Z)^{-1} C$ because the inverse of $(I-A Z)$ may not exist.

Since $\left.\operatorname{ker}\left(\cdot S^{*}\right)\right|_{\mathcal{U}_{2}^{\mathcal{N}}}$ is a DZ-invariant subspace, we have from the generalized Beurling-Lax theorem that there will be an isometric and causal transfer operator $\Sigma_{2}$ and an input sequence $\mathcal{K}$ such that $\left.\operatorname{ker}\left(\cdot S^{*}\right)\right|_{\mathcal{U}_{\mathcal{N}}^{\mathcal{N}}}=\mathcal{U}_{2}^{\mathcal{K}} \Sigma_{2}$. A state space realization for $\Sigma_{2}$ is easily derived from the realization for $S$, we just have to complete the local isometric matrices so that they become unitary:

$$
\mathbf{W}=\left[\begin{array}{cc}
A_{k} & C_{k} \\
B_{k} & D_{k} \\
B_{2 k} & D_{2 k}
\end{array}\right]
$$

$\Sigma_{2}$ shares $\mathcal{H}_{\circ}$ with $S$, and remarkably, also the defect space $\mathcal{K}_{o}^{\prime \prime}$

The isometric $S$ will hence possess a Darlington embedding iff its defect space is zero. This abstract condition translates to a concrete criterion, in the following important special case:

Theorem 7: The locally finite and causal isometric transfer operator $S$ will have a Darlington embedding if it has an isometric ue-stable realization.

Proof: An isometric realization has automatically a unit (and hence strictly nonsingular) observability Gramian $G_{0}$. If, on top of that, also $\ell_{A}<1$, then the embedding given by (33) actually produces

$$
\Sigma=\left[\begin{array}{c}
D \\
D_{2}
\end{array}\right]+\left[\begin{array}{c}
B \\
B_{2}
\end{array}\right] Z(I-A Z)^{-1} C
$$

with invertible $(I-A Z)^{-1}$ and its unitarity can be verified by direct calculation as before in Lemma 1 .

As in the LTI case, the Darlington synthesis is produced via a right coprime factorization for $S$ :

$$
S=\left[\begin{array}{ll}
I & 0
\end{array}\right] \Sigma
$$

and the algorithm is a version of the square root algorithm presented before.

\section{DARLINGTON EMBEDDING OF A CONTRACTIVE TIME-VARYING TRANSFER FUNCTION $S$}

We start out with a strictly contractive time-varying operator $S$ in state space form:

$$
S=D+B Z(I-A Z)^{-1} C
$$

and we assume, in addition, that the realization is ue-stable, $\ell_{A}=\sigma(A Z)<1$. We try to find a solution to the Darlington embedding problem, i.e. a $\Sigma$ such that $\Sigma$ is inner and embeds $S$ :

$$
\Sigma=\left[\begin{array}{cc}
S & \Sigma_{12} \\
\Sigma_{21} & \Sigma_{22}
\end{array}\right]
$$

Before formulating the realization theorem, we search for the solution by following an inductive approach partly inspired by [22]. The Darlington idea is to augment input and output spaces so that the resulting operator becomes unitary. A unitary operator will have a unitary realization, so let us try to construct a realization for $\Sigma$ of the form:

$$
\left[\begin{array}{ccc}
A & C & C_{2} \\
B & D & D_{12} \\
B_{2} & D_{21} & D_{22}
\end{array}\right]
$$

and which has the additional property that there is a state transformation $R$ which makes the transformed realization

$$
\left[\begin{array}{ccc}
R^{-1} A R^{(-1)} & R^{-1} C & R^{-1} C_{2} \\
B R^{(-1)} & D & D_{12} \\
B_{2} R^{(-1)} & D_{21} & D_{22}
\end{array}\right]
$$

unitary. The diagonal matrix $M=R R^{*}$ together with the entries $C_{2}$ and $D_{12}$ must then satisfy at least the following equations:

$$
\left\{\begin{array}{l}
A M^{(-1)} A^{*}+C C^{*}+C_{2} C_{2}^{*}=M \\
B M^{(-1)} B^{*}+D D^{*}+D_{12} D_{12}^{*}=I \\
A M^{(-1)} B^{*}+C D^{*}+C_{2} D_{12}^{*}=0
\end{array}\right.
$$

(The other entries will follow easily once these equations are solved, see further.) Observe that $C_{2}$ and $D_{12}$ can be eliminated further when $I-D D^{*}-B M^{(-1)} B^{*}$ is invertible, to produce the famous discrete time Riccati equation:

$$
\begin{aligned}
M= & A M^{(-1)} A^{*}+C C^{*}+\left(A M^{(-1)} B^{*}+C D^{*}\right) \\
& \times\left(I-D D^{*}-B M^{(-1)} B^{*}\right)^{-1}\left(B M^{(-1)} A^{*}+D C^{*}\right) .
\end{aligned}
$$


We shall not handle this equation directly, but the theory that we shall give produces a positive definite solution to it and gives conditions for the required invertibility as well. For an extensive study of Riccati equations and means to solve them directly, see the book [23]).

Remarkably, the solution to (37), if it exists, has a closed form for the positive definite $M$ and the related $C_{2}$ and $D_{12}$, which is found by a physical analysis of the properties of the Darlington embedding. The secret lays in the study of the 'future operator' connected to the embedded system which will have to be unitary when the Darlington embedding exists, and which we introduce now. The future operator of a system is found by decomposing inputs as $U=U_{p}+U_{f}$ and outputs as $Y=Y_{p}+Y_{f}$, according to the decomposition of spaces $\mathcal{X}_{2}=\mathcal{L}_{2} Z^{-1} \oplus \mathcal{U}_{2}$. When presented with the input $U_{p}$, the system $S$ produces a state given by $X$-a diagonal operator and the output $Y_{p}$, as given by the 'map of the past':

$$
\left[\begin{array}{ll}
X & Y_{p}
\end{array}\right]=U_{p}\left[\begin{array}{ll}
\mathcal{R} & S_{p}
\end{array}\right]
$$

in which $\mathcal{R}$ is the reachability map, and $S_{p}$, the map $U_{p} \mapsto Y_{p}$, a restriction of $S$ to signals 'in the past'. These expressions can be made concrete by decomposing the signals and operators in diagonals, see Appendix A for information and details. The state $X$ is in this case also a diagonal, which collects the states obtained at each time point $t$. Next, $X$ together with $U_{f}$ produce the output $Y_{f}$ according to the 'relations for the future':

$$
Y_{f}=\left[\begin{array}{ll}
X & U_{f}
\end{array}\right]\left[\begin{array}{c}
\mathcal{O} \\
S_{f}
\end{array}\right]
$$

in which

$$
\mathcal{O}=\left[C, A C^{(-1)}, A A^{(-1)} C^{(-2)}, \ldots\right]
$$

and

$$
S_{f}=\left.(\cdot S)\right|_{\mathcal{U}_{2}} \cdot
$$

The resulting maps look as in Fig. 5. Now we want to augment inputs and outputs, and allow for a bounded and boundedly invertible state transformation $R$, so that the resulting system becomes unitary. We call the additional inputs $U_{p a}$ and $U_{f a}$ and the state transformation $R$ and obtain the arrangement of Fig. 6 and the equations:

$$
\left[\begin{array}{ll}
Y_{f} & Y_{f a}
\end{array}\right]=\left[\begin{array}{lll}
X^{\prime} & U_{f} & U_{f a}
\end{array}\right]\left[\begin{array}{cc}
R^{-1} \mathcal{O} & R^{-1} \mathcal{O}_{a} \\
S_{f} & K_{12} \\
K_{21} & K_{22}
\end{array}\right]
$$

in which $\mathcal{O}_{a}, K_{12}, K_{21}$ and $K_{22}$ are new operators (an observability operator and three restriced transfer operators respect.) to be determined and whereby $X^{\prime}$ is the transformed state.

Suppose now that a solution does exist indeed, and let us compute the consequences. Unitarity requires at the least that

$$
\begin{aligned}
& {\left[\begin{array}{cc}
R^{-1} \mathcal{O} & R^{-1} \mathcal{O}_{a} \\
S_{f} & K_{12} \\
K_{21} & K_{22}
\end{array}\right] \cdot\left[\begin{array}{ccc}
\mathcal{O}^{*} R^{-*} & S_{f}^{*} & K_{21}^{*} \\
\mathcal{O}_{a} R^{-*} & K_{12}^{*} & K_{22}^{*}
\end{array}\right]} \\
& \quad=\left[\begin{array}{lll}
I & & \\
& I & \\
& & I
\end{array}\right] .
\end{aligned}
$$

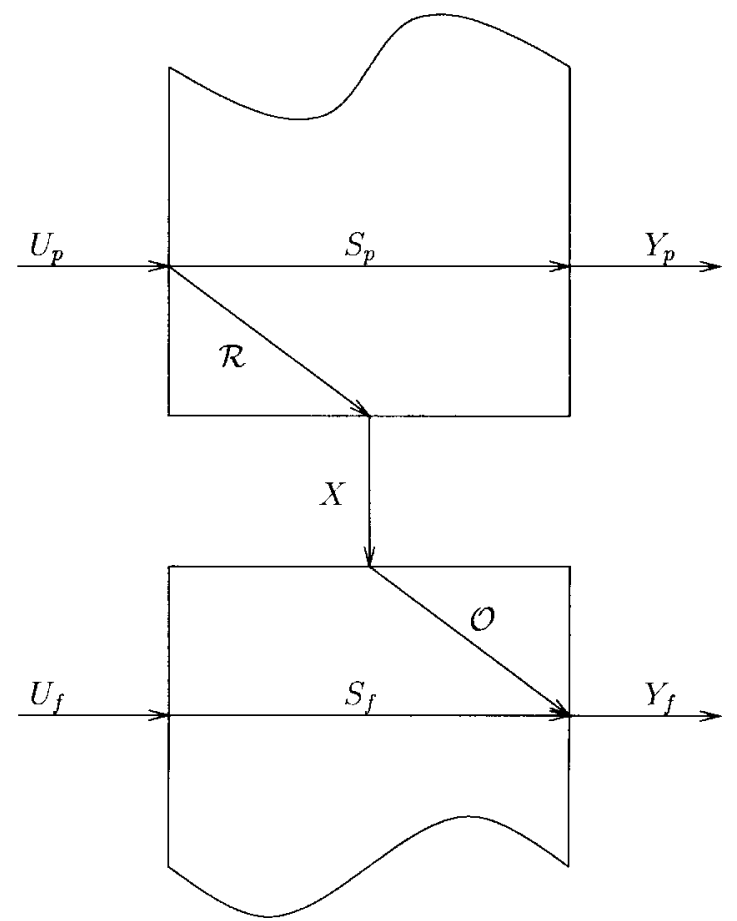

Fig. 5. The decomposition of the operator $S$ as a past and a future operator connected by the state.

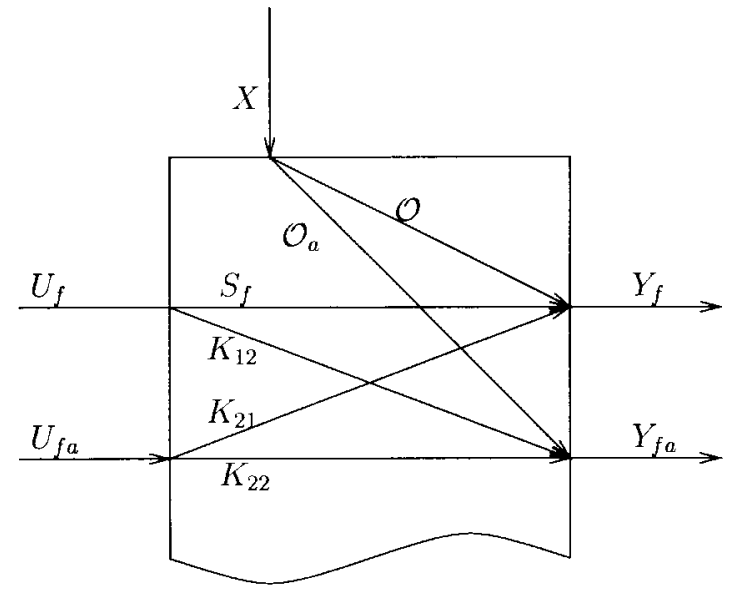

Fig. 6. The augmented system has a unitary map linking the state and the future input to the future output.

Hence, in particular, with $M=R R^{*}$

$$
\left\{\begin{array}{l}
\mathcal{O} \mathcal{O}^{*}+\mathcal{O}_{a} \mathcal{O}_{a}^{*}=M \\
\mathcal{O}_{f}^{*}+\mathcal{O}_{a} K_{12}^{*}=0 \\
S_{f} S_{f}^{*}+K_{12} K_{12}^{*}=I .
\end{array}\right.
$$

Since we assumed that $S$ is strictly contractive, the same holds true for $S_{f}$, because it is a restriction of $S$, and $I-S_{f} S_{f}^{*}$ is an invertible operator. Since

$$
K_{12} K_{12}^{*}=I-S_{f} S_{f}^{*}
$$

we may conclude that $K_{12}$ will be an invertible operator as well (it still has to be determined), and one must have

$$
\mathcal{O}_{a}=-\mathcal{O} S_{f}^{*} K_{12}^{-*}
$$


and

$$
\mathcal{O}_{a} \mathcal{O}_{a}^{*}=\mathcal{O} S_{f}^{*}\left(I-S_{f} S_{f}^{*}\right)^{-1} S_{f} \mathcal{O}^{*}
$$

Finally, we obtain the expression for $M$ :

$$
M=\mathcal{O}\left(I-S_{f} S_{f}^{*}\right)^{-1} \mathcal{O}^{*}
$$

in terms of originally known data!

Returning to the original embedding equations (37), we see (with some calculations, see [21]) that

$$
\left[\begin{array}{ccc}
R^{-1} A R^{(-1)} & R^{-1} C & R^{-1} C_{2} \\
B R^{(-1)} & D & D_{12}
\end{array}\right]
$$

will be isometric, if $M=R R^{*}$ given by (44) is invertible. In that case, the transformed $R^{-1} A R^{(-1)}$ will be ue-stable together with $A$, and the realization can be augmented pointwise to a locally unitary realization with a ue-stable transition operator. $M$ will be invertible iff the system is uniformly observable, i.e. iff $\mathcal{O}$ has a bounded inverse. If, on the other hand, the system is not uniformly observable, then, although a unitary embedding for the realization can be derived, it will usually not correspond to an inner embedding, because there will be nontrivial defect spaces. In that case, the transformed transition matrix $A=R^{-1} A R^{(-1)}$ will not be ue-stable. This defective case is considerably more difficult to analyze than the straight case where the given system $S$ is ue-stable and uniformly observable. Nonetheless, we have obtained a Darlington theorem for time-varying systems:

Theorem 8: If $S$ is a strictly contractive transfer operator with ue-stable locally finite realization which is uniformly observable, then $S$ possesses an inner embedding. If the ue-stable realization for $S$ is given by $\{A, B, C, D\}$, then the embedding has the unitary realization given by (36), in which the state transformation operator $R$ is obtained from $M=\mathcal{O}\left(I-S_{f} S_{f}^{*}\right)^{-1} \mathcal{O}^{*}$ as $M=R R^{*}$, which is also a positive definite solution of the Riccati equation (38).

Proof-Sketch: Suppose that $M$ is strictly positive definite, then it can be factored as $M=R R^{*}$ in which $R$ is a bounded and boundedly invertible operator. Then the state realization as given in Eqs. (36) turns out be orthogonal, which is verified by writing Eqs. (43) out in terms of the realizations. Since $R$ is bounded and boundedly invertible, the orthogonal realization given by (36) is ue-stable and is therefore, as stated in Lemma 1 , the realization of an inner transfer operator.

Square root algorithms to solve equations of the type (37) and the connected Riccati equation (38) have been known for a long time (for a literature survey, see [24]). Their interpretation as representative for factorization problems of the external or inner-outer type have been well documented also in the recent literature, see [22], [25]. In the context of the Darlington theory they get a particularly nice interpretation, since, as we have argued, the Darlington embedding problem reduces to a factorization problem. Conversely, to solve the Darlington embedding problem numerically, a square root algorithm would provide for the appropriate means. We derive the algorithm in Appendix B, further information on its numerical properties can be found in the book [21].

\section{DISCUSSION}

The focus of the present paper is the existence of the Darlington embedding, and we have seen that it is depended on the absence of a nontrivial 'defect' space in the input or output nullspace of the Hankel operator connected to the transfer operator of the system to be embedded. This is an unexpected conclusion, if one considers that the original problem statement as given by Darlington and the early researchers on the topic, Belevitch [6], Oono-Yasuura [26] and Youla [27], all considered it to be a problem in rational matrix factorization. The import of system theory for Darlington theory was most clearly realized by Vongpanitlerd and Anderson in their book [5], which gives a systematic account of the solution of classical circuit synthesis problems using state space formalism. However, only in the early 1970's some insight in the role played by external factorization and/or the Hankel operator (the two are closely related) in the problem started to dawn, and necessary and sufficient conditions for the existence of the Darlington or inner embedding appeared [2], [3], [19]. Later, a fairly complete picture for the time-varying case emerged along the same lines of reasoning, but the time-varying theory is considerably more delicate that the time invariant [21].

To conclude this paper, I want first to make the connection of the abstract embedding theory as presented in this paper with the classical cascade multiport synthesis problem, which is based on the factorization of the Chain Scattering Matrix, and has considerable interest in its own right. I conclude the discussion with some remarks concerning the practical use of Darlington theory. Darlington's original goal was to extend the cascade synthesis theory for a connecting network by factoring out a transmission zero at a point in the $s$ plane which was not located on the imaginary (for which an extraction theory already existed known as 'extraction of a Brune section'). This gave rise to the so-called 'Darlington section' [28], [29]. The cascade extraction theory can fairly easily be generalized to the LTI-multiport and even timevarying case. A recursive factorization theory as well as an equivalent cascade state space realization theory are available for each. In each instance, the most appealing way is to invoque the chain scattering matrix related to the embedding $\Sigma$, and either to factor it directly into elementary sections in the $s$-plane or the $z$-plane, or to consider its state space description in an algebraically reduced form. If the inner scattering operator is given by the input-output description

$$
\left[\begin{array}{ll}
b_{1} & a_{2}
\end{array}\right]=\left[\begin{array}{ll}
a_{1} & b_{2}
\end{array}\right]\left[\begin{array}{cc}
S & \Sigma_{12} \\
\Sigma_{21} & \Sigma_{22}
\end{array}\right]
$$

then the chain scattering description is defined by

$$
\left[\begin{array}{ll}
a_{2} & b_{2}
\end{array}\right]=\left[\begin{array}{ll}
a_{1} & b_{1}
\end{array}\right] \Theta .
$$

Theta will be well defined if $\Sigma_{12}$ or, equivalently, $\Sigma_{21}$ is boundedly invertible, and it is then given by

$$
\Theta=\left[\begin{array}{cc}
\Sigma_{12}-S \Sigma_{21}^{-1} \Sigma_{22} & -S \Sigma_{21}^{-1} \\
\Sigma_{21}^{-1} \Sigma_{22} & \Sigma_{21}^{-1}
\end{array}\right]=\left[\begin{array}{cc}
\Sigma_{12 *}^{-1} & -S \Sigma_{21}^{-1} \\
S_{*} \Sigma_{12 *}^{-1} & \Sigma_{21}^{-1}
\end{array}\right]
$$




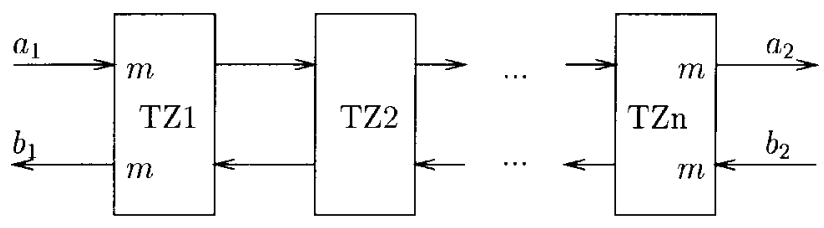

Fig. 7. A multiport cascade realization is obtained through the factorization of the Chain Scattering Matrix, whereby each section realizes one transmission zero.

We see that, in case of an LTI system, the poles of $\Theta$ are the conjugates of the poles of $\Sigma_{12}^{-1}$. When $\Sigma$ is inner, then $\Theta$ has important properties, some of which are:

- for the signature matrix:

$$
J=\left[\begin{array}{cc}
I_{m} & \\
& -I_{m}
\end{array}\right]
$$

$\Theta$ is para- $J$-unitary, meaning that:

$$
\Theta J \Theta_{*}=\Theta_{*} J \Theta=J
$$

(in the LTV-case, a similar property will hold but with a more complex signature operator $J$ );

- inside the unit disc of the complex plane, $\Theta(z)$ will be $J$-contractive, i.e.

$$
J-\Theta(z) J[\Theta(z)]^{*} \geq 0
$$

(remember that $*$ denotes the Hermitian transform). This relation follows from the relation (dropping the $z$-dependence):

$$
J-\Theta J \Theta^{*}=\left[\begin{array}{cc}
I & -S \Sigma_{21}^{-1} \\
0 & \Sigma_{21}^{-1}
\end{array}\right]\left[I-\Sigma \Sigma^{*}\right]\left[\begin{array}{cc}
I & 0 \\
-\Sigma_{21}^{-*} S^{*} & \Sigma_{21}^{-*}
\end{array}\right]
$$

obtained from the connection between $\Theta$ and $\Sigma$.

From the para- $J$-unitarity of $\Theta$, we see that the zeros of $\Theta$ (i.e. the poles of its inverse) are actually the conjugates of its poles, and vice versa. Hence, $\Theta$ shares zeros with $\Sigma_{12}$. This point may be exploited for algebraic benefit, by writing the embedding formula for $\Theta$ as

$$
\left[\begin{array}{ll}
I & S
\end{array}\right]=\left[\begin{array}{ll}
\Sigma_{12} & 0
\end{array}\right] \Theta^{-1}
$$

A cascade synthesis of $\Theta$ will now consist in extracting elementary sections from $\left[\Sigma_{12} 0\right]$, since in practical problems it is usually the transfer scattering matrix $\Sigma_{12}(z)$ that is given. It turns out that such a multiport, recursive extraction of elementary zeros using $J$-contractive, lossless sections is always possible [30], [31] and results in a cascade synthesis as shown in Fig. 7.

Alternatively, one can produce a formula with $\Sigma_{21}$, in which case an attractive embedding formula follows from the expression for

$$
\begin{aligned}
\Theta^{-1}=J \Theta^{*} J & =\left[\begin{array}{cc}
\Sigma_{12}^{-1} & \Sigma_{12}^{-1} S \\
\Sigma_{21}^{-*} S^{*} & \Sigma_{21}^{*}
\end{array}\right]: \\
\Theta^{-1}\left[\begin{array}{c}
S \\
-I
\end{array}\right] & =\left[\begin{array}{c}
0 \\
-\Sigma_{21}
\end{array}\right] .
\end{aligned}
$$

We shall exploit this formula in Appendix B to obtain a square root algorithm for the embedding in state space formalism.
The elementary sections for $\Theta$ to be used here have the following general forms - see in this respect the general work on multiport inner and Blaschke factors by Potapov [32], we give them for the sake of completeness:

- If the zero $a$ of $\Theta$ is located in $\mathrm{D}$ and $a \neq 0$ (multiport Darlington section):

$$
\Theta(a, V)(z)=I_{2 m}+\left(c_{a} \frac{z-a}{1-\bar{a} z}-1\right) J V V^{*}
$$

in which $c_{a}$ is a coefficient of modulus one and $V$ is a constant matrix such that $V^{*} J V=I_{k}$ for some integer $1 \leq k \leq m$ (the Smith-McMillan degree of such a section is exactly $k$ ).

- If the zero of $\Theta$ is located at $z=0$ (multiport Schur section):

$$
\Theta(0, V)(z)=I_{2 m}+\left(c_{0} z-1\right) J V V^{*}
$$

- if the zero of $\Theta$ is located at $z=e^{j \phi}$ (multiport Brune section):

$$
\Theta\left(e^{j \phi}, V\right)=I_{2 m}-r \frac{e^{j \phi}+z}{e^{j \phi}-z} J V V^{*}
$$

in which $r>0$ is a strictly positive real number and $V$ is a nonsingular, so called neutral matrix: $V^{*} J V=0$ (the columns of $V$ span a so called isotropic space, i.e. a space of elements that are all $J$-orthogonal to each other);

- if the zero of $\Theta$ is located at a point $a \in \mathbf{E}$ outside the unit disc:

$$
\Theta(a ; V)(z)=I_{2 m}-\left(c_{a} \frac{z-a}{1-\bar{a} z}-1\right) J V V^{*}
$$

in which $\left|c_{a}\right|=1$ and now $V^{*} J V=-I_{k}$ for some integer $1 \leq k \leq m$;

- and, finally, if the zero of $\Theta$ is at infinity:

$$
\Theta(\infty ; V)(z)=I_{2 m}-\left(c_{\infty} z^{-1}-1\right) J V V^{*}
$$

where $\left|c_{\infty}\right|=1$ and $V^{*} J V=-I_{k}$ for some integer $k$ such that $1 \leq k \leq m$.

The network-theoretical literature has been very much concerned with the properties of these various sections and certain cascades of them. If a strict, minimal degree synthesis is desired, then at each elementary extraction, the degree should go down by a number equal to the degree of the section that is being extracted, $k$ for the sections given above. However, it is also possible to extract sections without the degree reduction, but respecting the passivity of the remainder circuit. In that case, correct approximating networks are obtained when appropriate interpolation conditions are satisfied. There is an extensive literature on the properties of the approximations obtained, it is of interest for circuit reduction in the VLSI modeling context [33].

I finish with a brief discussion of three important practical problems which have found elegant solutions thanks to the generalized Darlington theory, but whose discussion falls outside the context of this paper. 
Broadband Matching: Darlington theory provides the key ingredient in broadband matching theory, as it succeeds in representing both the source impedance and the load impedance as a Darlington connecting network, and the broadband matching problem then is converted to the design of a connecting section. See the literature for various approaches [34], [35], [36].

Minimal Algebraic Computations and Canonic Representations of Systems: Darlington synthesis leads to numerical realizations of operators with minimal algebraic complexity, see [21] for an extensive discussion of this topic. The key ingredient here is the fact that a unitary representation can be realized with a number of elementary rotations which is algebraically minimal, i.e. equal to the number of free algebraic parameters. Although other methods of obtaining algebraically minimal realizations are possible, the one based on unitary realizations and elementary rotations is particularly attractive because it combines algebraic minimality with numerical operations that have minimal error propagation. Algebraic minimality can be very important at least for two reasons. One is that a variation of a parameter in an algebraically minimal realization will not change the class of the realization (for example: a lossless circuit will stay lossless). This fact has been extensively exploited by the very elegant realization theory for Wave Digital Filters discovered and pioneered by A. Fettweiss [37]. The second reason is that algebraically minimal realizations can be build in such a way that they form a continuum for all possible realizations of a given degree and hence can be used for circuit optimalization by continuous variation of parameters.

Inversion of Systems: Recently, numerical inversion theory of systems of equations has been extended to handle a pretty general type of infinite systems which are described with a finite set of parameters. The class for which the best solution have been obtained consists of systems which are time invariant for $t \rightarrow \pm \infty$ but are varying in between. It appears that inner-outer factorization, the main mechanism for Darlington synthesis, again plays the key role in solving the inversion problem.

\section{APPENDIX A}

\section{Past-Future Decomposition of a Causal Operator $S$}

The concrete representation of operators such as $S_{p}, \mathcal{R}, \mathcal{O}$ and $S_{f}$ in (39) and (40) hinge on the choice of bases in the input and output spaces of these operators. In our case, the handiest choice for the input space $\mathcal{U}$ and the output space $\mathcal{Y}$ are decompositions in diagonals as

$$
U=\sum_{i=-\infty}^{\infty} U_{i} Z^{i}, \quad Y=\sum_{i=-\infty}^{\infty} Y_{i} Z^{i}
$$

in which $U_{i}$ is the $i$-th diagonal of $U$, properly positioned. Let us also represent the operator $S$ by its diagonal decomposition:

$$
S=\sum_{k=0}^{\infty} S_{k} Z^{k}
$$

This is a basis decomposition in which the constituting elements are diagonal operators. Hence, viewed as series, $U$ and $Y$ can now be represented by series of diagonals:

$$
\begin{aligned}
\tilde{U} & =\left[\ldots, U_{-2}, U_{-1}, U_{0}, U_{1}, U_{2}, \ldots\right] \\
\tilde{Y} & =\left[\ldots, Y_{-2}, Y_{-1}, Y_{0}, Y_{1}, Y_{2}, \ldots\right]
\end{aligned}
$$

The operator $S_{p}$ maps $\tilde{U}_{p}=\left[\ldots, U_{-2}, U_{-1}\right]$ to $\tilde{Y}_{p}=$ $\left[\ldots, Y_{-2}, Y_{-1}\right]$ and, taking notice of the rule $Z^{-k} S_{\ell}=$ $S_{\ell}^{(k)} Z^{-k}$, it will be represented by

$$
\tilde{S}_{p}=\left[\begin{array}{cccc}
\ddots & \vdots & \vdots & \vdots \\
\cdots & S_{0}^{(3)} & S_{1}^{(3)} & S_{2}^{(3)} \\
\cdots & 0 & S_{0}^{(2)} & S_{1}^{(2)} \\
\cdots & 0 & 0 & S_{0}^{(1)}
\end{array}\right]
$$

Likewise, $S_{f}$ maps $U_{f}$ to $Y_{f}$, and will also be represented, in the diagonal bases chosen, by an operator $\tilde{S}_{f}$ consisting of diagonals. Using the rule $Z^{k} S_{\ell}=S_{\ell}^{(-k)} Z^{k}$, it will be given by:

$$
\tilde{S}_{f}=\left[\begin{array}{cccc}
S_{0} & S_{1} & S_{2} & \cdots \\
0 & S_{0}^{(-1)} & S_{1}^{(-1)} & \cdots \\
0 & 0 & S_{0}^{(-2)} & \ldots \\
\vdots & \vdots & \vdots & \ddots
\end{array}\right]
$$

The connecting operators $\mathcal{R}$ and $\mathcal{O}$ generate the state $X$ through the action of the Hankel operator, which, as expected, factors out, since $S=D+B C^{(-1)} Z+B A^{(-1)} C^{(-2)} Z^{2}+$ $B A^{(-1)} A^{(-2)} C^{(-3)} Z^{3}+\cdots$, as

$$
\begin{aligned}
& \tilde{H}=\left[\begin{array}{cccc}
\vdots & \vdots & \vdots & \cdot \\
S_{3}^{(3)} & S_{4}^{(3)} & S_{5}^{(3)} & \cdots \\
S_{2}^{(2)} & S_{3}^{(2)} & S_{4}^{(2)} & \ldots \\
S_{1}^{(1)} & S_{2}^{(1)} & S_{3}^{(1)} & \ldots
\end{array}\right] \\
& =\left[\begin{array}{c}
\vdots \\
B^{(3)} A^{(2)} A^{(1)} \\
B^{(2)} A^{(1)} \\
B^{(1)}
\end{array}\right]
\end{aligned}
$$

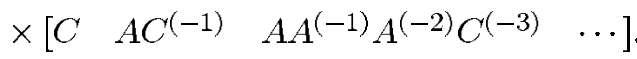

Hence, 'concrete' representations for $\mathcal{R}$ and $\mathcal{O}$ in the diagonal algebra are given by

$$
\begin{aligned}
\mathcal{R} & =\left[\begin{array}{c}
\vdots \\
B^{(3)} A^{(2)} A^{(1)} \\
B^{(2)} A^{(1)} \\
B^{(1)}
\end{array}\right] \\
\mathcal{O} & =\left[\begin{array}{llll}
C & A C^{(-1)} & A A^{(-1)} A^{(-2)} C^{(-3)} & \cdots
\end{array}\right] .
\end{aligned}
$$

It should be clear, then, that $X$ is indeed given by the diagonal:

$$
X=\cdots+U_{-3} B^{(3)} A^{(2)} A^{(1)}+U_{-2} B^{(2)} A^{(1)}+U_{-1} B^{(1)} .
$$




\section{APPENDIX B}

\section{SQUARE ROOT ALGORITHM FOR THE DARLINGTON EMBEDDING}

The square root algorithm for the Darlington embedding as derived by [22] can be interpreted as a state space expression of the Darlington embedding equation (49), which itself is a special instance of an inner-outer factorization equation. If the original scattering operator $S$ has a realization $\{A, B, C, D\}$, then the outer spectral factor $\Sigma_{21}$ of $\left(I-S^{*} S\right)$ will share the $\{A, C\}$ with $S$, and will have $B_{2}$ and $D_{21}$ determined by the condition that there should exist a bounded and boundedly invertible state transformation operator $R$ such that

$$
\Sigma_{a}=\left[\begin{array}{lll}
R & & \\
& I & \\
& & I
\end{array}\right]\left[\begin{array}{cc}
A & C \\
B & D \\
B_{2} & D_{21}
\end{array}\right]\left[\begin{array}{cc}
R^{-(-1)} & \\
& I
\end{array}\right]
$$

is co-isometric (this is the dual case of the factorization case considered in the main text of this paper, for good reason, see the brief discussion at the end of this section). Expressing the embedding (49) in terms of these diagonal state space operators now takes the form

$$
\Theta\left[\begin{array}{cc}
R A & R C \\
B & D \\
0 & -I
\end{array}\right]=\left[\begin{array}{cc}
R^{(-1)} & 0 \\
0 & 0 \\
-B_{2} & -D_{21}
\end{array}\right]
$$

in which $\Theta$ is actually a $J$-unitary realization matrix for the anticausal transfer function $\Theta^{-1}$. These equations form the basis of the square-root algorithm to compute the embedding. They state that, given the original state space realization for $S$ and the value of $R_{k}$ at a given point $k$ in the recursion, one finds $R_{k+1}, B_{2 k}$ and $D_{21 k}$ by annihilating the middle rows of

$$
\left[\begin{array}{cc}
R_{k} A_{k} & R_{k} C_{k} \\
B_{k} & D_{k} \\
0 & -I
\end{array}\right]
$$

using a $J$-unitary transformation matrix, with

$$
J=\left[\begin{array}{ccc}
I & & \\
& I & \\
& & -I
\end{array}\right],
$$

to produce

$$
\left[\begin{array}{cc}
R_{k+1} & 0 \\
0 & 0 \\
-B_{2, k+1} & -D_{21, k+1}
\end{array}\right] .
$$

The existence of the Darlington embedding actually insures the existence of the transformation matrix (if it had been unitary, it would always exist, but $J$-unitarity is a different case). For further details on implementation and numerical properties, see [21].

The square-root algorithm shown here computes an outer companion $\Sigma_{21}$ and a causal embedding $\Theta$ to $S$. This corresponds to the traditional Darlington embedding method whereby a transfer scattering operator $\Sigma_{12}$ is first converted to an input scattering operator $S$, which is then further realized using cascaded sections with the correct transmission zeros. The dual factorization method given in Section VIII is in a sense more interesting: it proceeds directly with the factorization of the given $\Sigma_{12}$, and produces both $\Theta$ and $S$ doing so.

\section{ACKNOWLEDGMENT}

The author wishes to thank D. Pik of the Department of Mathematics and Computer Sciences of the the Free University of Amsterdam, Amsterdam, The Netherlands, for his suggestions, comments and proofreading of the manuscript.

\section{REFERENCES}

[1] S. Darlington, "Synthesis of reactance 4-poles which produce a prescribed insertion loss characteristics," J. Math. Phys., vol. 18, pp. 257-355, 1939.

[2] P. Dewilde, "Roomy scattering matrix synthesis," Tech. Rep., Dept. of Mathematics, Univ. of California, Berkeley, 1971.

[3] D. Arov, "On the Darlington method in the theory of dissipative systems," Dokl. Akad. Nauk USSR, vol. 201, no. 3, pp. 559-562, 1971.

[4] H. Helson, Lectures on Invariant Subspaces. New York: Academic, 1964.

[5] B. Anderson and S. Vongpanitlerd, Network Analysis and Synthesis. Englewood Clifffs, NJ: Prentice Hall, 1973.

[6] V. Belevitch, "Elementary applications of the scattering formalism to network design," IRE Trans. Circuit Theory, vol. CT-3, pp. 97-104, June 1956.

[7] $\frac{}{1968 .}$, Classical Network Theory. San Francisco, CA: Holden Day,

[8] J. Neirynck and P. V. Bastelaer, "La synthèse des filtres par la factorization de la matrice de transfert," Rev. MBLE, vol. X, no. 1, pp. 5-32, 1967.

[9] D. Youla, "Cascade synthesis of passive $n$-ports," Polytechnic Inst. of Brooklyn, NY, Tech. Rep., June 1965.

[10] V. B. P. Dewilde and R. Newcomb, "On the problem of degree reduction of a scattering matrix by factorization," J. Franklin Inst., vol. 291, no. 5, pp. 387-401, May 1971.

[11] P. Dewilde, "Cascade scattering matrix synthesis," Ph.D. dissertation, Stanford University, CA, June 1970.

[12] W. Rudin, Real and Complex Analysis. New York: McGraw-Hill, 1966.

[13] K. Hoffman, Banach Spaces of Analytic Functions. Englewood Cliffs, NJ: Prentice-Hall, 1962.

[14] L. C. D. C. Youla and H. Carlin, "Bounded real scattering matrices and the foundation of linear passive network theory," IRE Trans., vol. CT-4, no. 1, pp. 102-124, Mar. 1959; corrections, Ibid., p. 317, Sept. 1959.

[15] D. Youla and P. Tissi, " $n$-port synthesis via reactance extraction-Part I," IEEE Int. Conf. Rec., 1966, vol. 14, no. 7, pp. 183-205.

[16] P. M. P. and N. Wiener, "The prediction theory of multivariable stochastic processes," Acta Math., vol. 98, pp. 111-150, 1957; and Acta Math., vol. 99, pp. 93-137, 1958.

[17] G. Szegö, "Beitrage zur Theorie der Toeplitzen Formen (Ersten Mitteilung)," Math. Zeit, vol. 6, 1920.

[18] R. Saeks, "The factorization problem-A survey," Proc. IEEE,vol. 64 pp. 90-95, Jan. 1976.

[19] P. Dewilde, "Input-output description of roomy systems," SIAM J. Contr. Opt., vol. 14, pp. 712-736, July 1976.

[20] R. Kalman, P. Falb, and M. Arbib, "Topics in mathematical system theory," Int. Series in Pure and Applied Math. New York: McGrawHill, 1970.

[21] P. Dewilde and A.-J. van der Veen, Time-Varying Systems and Computations. Amsterdam, The Netherlands, Kluwer, 1998.

[22] A. van der Veen, "Time-varying system theory and computational modeling: Realization, approximation, and factorization," Ph.D. dissertation, Delft Univ. of Technology, Delft, The Netherlands, June 1993.

[23] P. Lancaster and L. Rodman, Algebraic Riccati Equations. Oxford, U.K.: Oxford Univ. Press, 1995.

[24] M. Morf and T. Kailath, "Square-root algorithms for least-squares estimation," IEEE Trans. Automat. Contr., vol. 20, no. 4, pp. 487-497, 1975 . 
[25] A. Halanay and V. Ionescu, "Time-Varying discrete linear systems," Operator Theory: Advances and Applications. Germany: Birkhäuser, vol. 68, 1994.

[26] Y. Oono and K. Yasuura, "Synthesis of finite passive $2 n$-terminal networks with prescribed scattering matrices," Mem. Fac. Eng. Kyushu Univ., vol. 14, no. 2, pp. 125-177, May 1954.

[27] D. Youla, "A new theory of cascade synthesis," IRE Trans. Circuit Theory, vol. 8, pp. 244-260, Sept. 1961; also, "Correction," IRE Trans. Circuit Theory, vol. CT-13, pp. 90-91, Mar. 1966.

[28] A. Belevitch, Classical Network Theory. San Francisco, CA: Holden Day, 1968.

[29] R. Newcomb, Linear Multiport Synthesis New York: McGraw Hill, 1966.

[30] P. Dewilde, "Cascade scattering matrix synthesis," Ph.D. dissertation, Stanford University, Stanford, CA, 1970.

[31] P. Dewilde and H. Dym, "Lossless inverse scattering, digital filters, and estimation theory," IEEE Trans. Inform. Theory, vol. 30, pp. 644-662, July 1984.

[32] V. Potapov, "The multiplicative structure of $J$-contractive matrix functions," Amer. Math. Soc. Transl. Ser. 2, vol. 15, pp. 131-243, 1960.

[33] P. Dewilde and Z.-Q. Ning, Models for Large Integrated Circuits, Boston: Kluwer, 1990

[34] J. W. Helton, "Broadbanding: Gain equalization directly from data," IEEE Trans. Circuits Syst., vol. CAS-28, Dec. 1981.

[35] H. Carlin and J.-C. C. Wu, "Amplitude selectivity versus constant delay in minimum-phase lossless filters," IEEE Trans. Circuits Syst., vol. CAS-23, pp. 447-455, July 1976.

[36] Y. Chan and E. Kuh, "A general matching theory and its applications to tunnel diode amplifiers," IEEE Trans. Circuit Theory, vol. CT-13, no. 1, pp. 6-18, 1966

[37] A. Fettweiss, "Digital filter structures related to classical filter networks," A.E.U., vol. 25, pp. 79-89, 1971

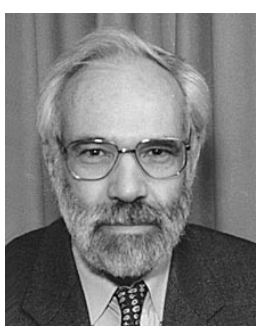

Patrick Dewilde (S'69-M'73-SM'81-F'82) received the degree of electrical engineering from the University of Leuven in 1966, the License in Mathematics from the Belgian Central Examination Commission in 1968 and the Ph.D. degree in electrical engineering from Stanford University in 1970 .

He has held various research and teaching positions at the University of California in Berkeley, the University of Lagos in Nigeria and the University of Leuven, Leuven, Belgium. In 1977 he became full professor of Electrical Engineering at the Technical University of Delft (the Netherlands). In 1981 he was named Fellow of the IEEE for his work on Scattering Theory. His research interests include the design of integrated circuits (VLSI) especially in the area of signal processing, large scale computational problems, theoretical topics in system theory and signal processing, and information management. He has been a project leader of major European projects in Microelectronics. The NELSIS design system, which pioneered a unique design information management methodology, was developed under his direction. In 1993, he became the Scientific Director of DIMES, the Delft Institute of Microelectronics and Submicron Technology, which employs more than 300 researchers active in advanced Microelectronics. He is the author of a large number of scientific publications, a book entitled Large Scale Modeling of Integrated Circuits (Kluwer, 1988) and one entitled Time Varying Systems and Computations (Kluwer, 1998).

Dr. Dewilde was elected as a regular member of the Dutch Royal Academy of Science in 1993. 